\title{
Small points on rational subvarieties of tori
}

Francesco Amoroso and Evelina Viada

\begin{abstract}
Let $V$ be a subvariety of a torus defined over the rational numbers. We study the distribution of points of small Weil's height on $V$. We simplify the proof and we improve previous results by the first author and S. David. We obtain a totally explicit version of a generalized Dobrowolski result on the Lehmer problem.
\end{abstract}

Mathematics Subject Classification (2010). 11G50, 11K60.

Keywords. Heights, Lehmer's problem.

\section{Introduction}

In this article we study the distribution of the small points of proper subvarieties of the torus $\mathbb{G}_{\mathrm{m}}^{n}$ defined over $\mathbb{Q}$. For $n=1$, the problem corresponds to finding lower bounds for the Weil height of an algebraic number. Let $\alpha$ be a non-zero algebraic number of degree $D$ which is not a root of unity. Lehmer (see [Leh]) asked whether there exists an absolute constant $c>0$ such that $h(\alpha) \geq \frac{c}{D}$. The best known result in this direction is Dobrowolski's result ([Dob]): if $D>1$,

$$
h(\alpha) \geq \frac{c}{D}\left(\frac{\log D}{\log \log D}\right)^{-3}
$$

for some absolute constant $c>0$. Dobrowolski's theorem was generalized to $\mathbb{Q}$ irreducible subvarieties $V \subseteq \mathbb{G}_{\mathrm{m}}^{n}$ in a series of articles by David and the first author. They prove the Generalized Dobrowolski Bound stated below. Their proofs are long and involved. Mainly, they need an intricate descent argument, hard to read by non specialists. This descent has been used in several occasions by other authors. Our first achievement in this paper is a simple and short proof of an explicit and improved version of the Generalized Dobrowolski Bound. More precisely, we generalize this statement describing the distribution of small points for different invariants. In addition we improve some bounds in the applications.

We fix the usual embedding of $\mathbb{G}_{\mathrm{m}}^{n}$ in $\mathbb{P}^{n}$ given by $\boldsymbol{x}=\left(x_{1}, \ldots, x_{n}\right) \mapsto\left(1: x_{1}\right.$ : $\left.\cdots: x_{n}\right)$. For a set $S \subseteq \mathbb{G}_{\mathrm{m}}^{n}$, we denote by $\bar{S}$ the Zariski closure of $S$ in $\mathbb{G}_{\mathrm{m}}^{n}$. On $\mathbb{P}^{n}$ 
we consider the Weil logarithmic absolute height, denoted by $h(\cdot)$. Given $\varepsilon>0$ we denote by $S(\varepsilon)$ the set of $\alpha \in S \cap \mathbb{G}_{\mathrm{m}}(\overline{\mathbb{Q}})$ of height $\leq \varepsilon$. A variety $V \subseteq \mathbb{G}_{\mathrm{m}}^{n}$ is the intersection of $\mathbb{G}_{\mathrm{m}}^{n}$ with a variety of $\mathbb{P}^{n}$ defined over $\overline{\mathbb{Q}}$. Note that the varieties which appear in this paper are not necessarily irreducible or equidimensional. However we consider only proper subvarieties of $\mathbb{G}_{\mathrm{m}}^{n}$, therefore we say subvariety of $\mathbb{G}_{\mathrm{m}}^{n}$ for proper subvariety of $\mathbb{G}_{\mathrm{m}}^{n}$. We define the essential minimum $\hat{\mu}^{\text {ess }}(V)$ of $V$ as the infimum of the set of $\varepsilon>0$ such that $V(\varepsilon)$ is Zariski-dense in $V$. We say that $B \subset \mathbb{G}_{\mathrm{m}}^{n}$ is torsion if it is a translate of a subtorus by a torsion point. The Kronecker theorem for points and the Bogomolov conjecture (Zhang [Zha]) for varieties of positive dimension yield

$$
\hat{\mu}^{\text {ess }}(V)>0 \text { if and only if } V \text { is not a union of torsion varieties. }
$$

According to different geometric and arithmetic assumptions, we relate $\hat{\mu}^{\text {ess }}(V)$ to different invariants of $V$, proving essentially sharp effective versions of (1.1). Lehmer's conjecture can be seen as a sharp effective version of (1.1) for points. The Generalized Dobrowolski Bound is a quasi optimal effective version of (1.1) for varieties defined over $\mathbb{Q}$ of arbitrary dimension. For varieties over arbitrary number fields which are not union of translates of subtori we speak of Effective Bogomolov. This case has been treated in our previous work [Amo-Via]. Note that there are intersections between the two problems, namely for varieties over $\mathbb{Q}$ which are not translates. Therefore an interesting new case treated in this work, is the one of translates defined over $\mathbb{Q}$ and specially the case of 0 -dimensional varieties consisting of the conjugates of a non-torsion point $\alpha \in \mathbb{G}_{\mathrm{m}}^{n}(\overline{\mathbb{Q}})$. Naturally the Galois group plays a key role in this work.

Let us introduce relevant invariants of a proper projective subvariety $V \subseteq \mathbb{P}^{n}$. The obstruction index $\omega(V)$ is the minimum degree of a hypersurface $Z$ containing $V$. Define $\delta(V)$ as the minimal degree $\delta$ such that $V$ is, as a set, the intersection of hypersurfaces of degree $\leq \delta$. Finally, define $\delta_{0}(V)$ as the minimal degree $\delta_{0}$ such that there exists an intersection $X=Z_{1} \cap \cdots \cap Z_{r}$ of hypersurfaces $Z_{j}$ of degree $\leq \delta_{0}$ such that any $\overline{\mathbb{Q}}$-irreducible component of $V$ is a $\overline{\mathbb{Q}}$-irreducible component of $X$. In Corollary 2.3 we prove that if $V$ is defined over $\mathbb{Q}$, we can choose the above hypersurfaces $Z, Z_{1}, \ldots, Z_{r}$ also defined over $\mathbb{Q}$.

The following effective version of (1.1) is proved in [Amo-Dav] for $\operatorname{dim} V=0$, in [Amo-Dav] for codim $V=1$ and in [Amo-Dav] for varieties of arbitrary dimension.

Generalized Dobrowolski Bound. Let $V$ be a subvariety of $\mathbb{G}_{\mathrm{m}}^{n}$ defined over $\mathbb{Q}$ of codimension $k$. Let us assume that $V$ is not contained in any union of proper torsion varieties.

Then, there exist two positive constants $c(n)$ and $\kappa(k)=(k+1)(k+1) !^{k}-k$ such that

$$
\hat{\mu}^{\text {ess }}(V) \geq \frac{c(n)}{\omega(V)}(\log 3 \omega(V))^{-\kappa(k)} .
$$


To recover a slightly weaker version of Dobrowolski's theorem it is sufficient to take $V$ equal to the set of conjugates of the algebraic number $\alpha$.

For a subvariety $V$ of $\mathbb{G}_{\mathrm{m}}^{n}$, we denote by $V^{*}$ the complement in $V$ of the union of the torsion varieties $B \subseteq V$. By (1.1) the minimum of the height on $V^{*}(\overline{\mathbb{Q}})$ is $>0$. In $\left[\right.$ Amo-Dav] is proved that for a $\mathbb{Q}$-irreducible $V$ and $\boldsymbol{\alpha} \in V^{*}(\overline{\mathbb{Q}})$

$$
h(\boldsymbol{\alpha}) \geq \frac{c(n)}{\delta(V)}(\log 3 \delta(V))^{-\kappa(n)} .
$$

where $c(n)>0$ is not computed and where $\kappa(n) \approx n^{n^{2}}$ is as above. Notice that this lower bound implies (1.2), with a possible worse exponent on the remainder term. To see that, apply (1.3) to a hypersurface $Z \supseteq V$ defined over $\mathbb{Q}$ and of degree $\omega(V)$.

For $n=1$ Dobrowolski's result remains the best known. In order to simplify the exposition and the computation of the constants we prefer to assume $n \geq 2$. Our first achievement is a simple and short proof of an explicit and improved version of (1.3):

Theorem 1.1. Let $V \subseteq \mathbb{G}_{\mathrm{m}}^{n}$ be a $\mathbb{Q}$-irreducible variety of dimension $d$. Then, for any $\boldsymbol{\alpha} \in V^{*}(\overline{\mathbb{Q}})$

$$
h(\boldsymbol{\alpha}) \geq \delta(V)^{-1}\left(935 n^{5} \log \left(n^{2} \delta(V)\right)\right)^{-(d+1)(n+1)^{2}} .
$$

In short, the exponent $\kappa(n)$ on the remainder term is improved by one exponential. In addition the constant $c(n)$ is computed. This could be useful in possible applications. However, the most interesting aspect remains the simplicity of the new method. We avoid the technical descent argument and the generalization of Philippon zero's estimate used in [Amo-Dav]. This new method could find other applications, as for instance in the context of the Relative Lehmer Problem, where methods similar to the ones of David and the first author are used (see [Del]).

To be able to use a conclusive geometric induction similar to the one presented in [Amo-Via] we first need to produce a new sharp lower bound for $\hat{\mu}^{\text {ess }}(V)$ in terms of $\delta_{0}(V)$ for varieties which are not union of torsion varieties.

Theorem 1.2. Let $V$ be a subvariety of $\mathbb{G}_{\mathrm{m}}^{n}$ of codimension $k$, defined and irreducible over $\mathbb{Q}$. Assume that $V$ is not a union of torsion varieties. Let

$$
\theta_{0}=\delta_{0}(V)\left(52 n^{2} \log \left(n^{2} \delta_{0}(V)\right)\right)^{(n+1)(k+1)} .
$$

Then there exists a hypersurface $Z$ defined over $\mathbb{Q}$ of degree at most $\theta_{0}$ which does not contain $V$ and such that

$$
V\left(\theta_{0}^{-1}\right) \subseteq V \cap Z
$$

This theorem is the arithmetic counterpart to [Amo-Via], Theorem 2.1. On one side, $V$ has to be defined over $\mathbb{Q}$, assumption not necessary in [Amo-Via]. On the 
other side $V$ can be a union of translates of torsion varieties by non-torsion points, situation to avoid in [Amo-Via]. Despite some similarity, the methods used in other works are not sufficient to prove this theorem. As in [Amo-Via], we first produce an inequality involving some parameters, $\hat{\mu}^{\text {ess }}(V)$ and the Hilbert functions of two varieties related to $V$ (Theorem 3.1). Some ingredients of the proof of Theorem 3.1 come from [Amo-Dav]. The main difference is the following. In the quoted paper, using Siegel's lemma, the authors construct one auxiliary function vanishing on $V$ and then they extrapolate to show that the obstruction index of $[p] V$ is small. Here we use Siegel's lemma in its full power and we find a family of linearly independent auxiliary functions vanishing on $V$. Then, we extrapolate at $[p] V$ for each auxiliary function. We don't use an interpolation determinant, as in [Amo-Via], because the problem is not symmetric. Another important difference is that, to decode the diophantine information in Theorem 3.1 it is not sufficient to use the estimates for the Hilbert function due to M. Chardin and P. Philippon [Cha-Phi], like we do in [Amo-Via]. In the present situation we need a refinement of their results which is proved in the appendix of this article by M. Chardin and P. Philippon. A further subtle point is to control the behavior of $\delta_{0}$ under the action of groups (Proposition 2.7). The final geometric induction allows us to prove the main result of this article:

Theorem 1.3. Let $V_{0} \subseteq V_{1}$ be subvarieties of $\mathbb{G}_{\mathrm{m}}^{n}$, defined over $\mathbb{Q}$, of codimensions $k_{0}$ and $k_{1}$ respectively. Assume that $V_{0}$ is $\mathbb{Q}$-irreducible. Let

$$
\theta=\delta\left(V_{1}\right)\left(935 n^{5} \log \left(n^{2} \delta\left(V_{1}\right)\right)\right)^{\left(k_{0}-k_{1}+1\right)\left(k_{0}+1\right)(n+1)} .
$$

Then,

- either there exists a $\mathbb{Q}$-irreducible $B$ union of torsion varieties such that $V_{0} \subseteq$ $B \subseteq V_{1}$ and $\delta_{0}(B) \leq \theta$,

- or there exists a hypersurface $Z$ defined over $\mathbb{Q}$ of degree at most $\theta$ such that $V_{0} \nsubseteq Z$ and $V_{0}\left(\theta^{-1}\right) \subseteq Z$.

In Section 5, we show how to deduce Theorem 1.1. In addition we prove some corollaries. Combining Theorem 1.1 with the estimate on the sum of the degrees of the maximal torsion varieties of $V$ ([Amo-Via], Corollary 5.3), we can give the following complete description of the small points of $V$.

Corollary 1.4. Let $V \subseteq \mathbb{G}_{\mathrm{m}}^{n}$ be a $\mathbb{Q}$-irreducible variety of dimension $d$. Let

$$
\theta=\delta(V)\left(935 n^{5} \log \left(n^{2} \delta(V)\right)\right)^{(d+1)(n+1)^{2}} .
$$

Then

$$
\overline{V\left(\theta^{-1}\right)}=B_{1} \cup \cdots \cup B_{t},
$$


where $B_{1}, \ldots, B_{t}$ are the maximal torsion varieties of $V$. In addition, $\delta_{0}\left(B_{j}\right) \leq \theta$ and

$$
\sum_{j=1}^{t} \theta^{\operatorname{dim}\left(B_{j}\right)} \operatorname{deg}\left(B_{j}\right) \leq \theta^{n} .
$$

A direct application of Theorem 1.3 allows us to show

Corollary 1.5. Let $V \subseteq \mathbb{G}_{\mathrm{m}}^{n}$ be a $\mathbb{Q}$-irreducible subvariety of codimension $k$ which is not contained in any union of proper torsion varieties. Then

$$
\hat{\mu}^{\text {ess }}(V) \geq \omega(V)^{-1}\left(935 n^{5} \log \left(n^{2} \omega(V)\right)\right)^{-k(k+1)(n+1)} .
$$

As mentioned, also Theorem 1.1 implies a similar but less sharp lower bound for the essential minimum, where the exponent on the remainder term is $n(n+1)^{2}$ instead of the better $k(k+1)(n+1)$.

An important application of Corollary 1.5 is a lower bound for the product of the heights of multiplicatively independent algebraic numbers. For instance, this kind of result is used by Bombieri, Masser and Zannier to show the finiteness of the intersection of a transverse curve with the union of all subtori of codimension two [Bom-Mas-Zan]. From Corollary 1.5 we deduce the following refined version of [Amo-Dav], Theorem 1.6:

Corollary 1.6. Let $\alpha_{1}, \ldots, \alpha_{n}$ be multiplicatively independent algebraic numbers in a number field $K$ of degree $D=[K: \mathbb{Q}]$. Then

$$
h\left(\alpha_{1}\right) \ldots h\left(\alpha_{n}\right) \geq D^{-1}\left(1050 n^{5} \log (3 D)\right)^{-n^{2}(n+1)^{2}} .
$$

The dependence on $\delta$ (or $\omega$ ) of our results is essentially sharp. However, the dependence in the dimension $n$ of the ambient variety remains mysterious. One could conjecture that for all $\mathbb{Q}$-irreducible linear subvarieties $V \subseteq \mathbb{G}_{\mathrm{m}}^{n}$ and for all $\boldsymbol{\alpha} \in V^{*}(\overline{\mathbb{Q}})$ we had $h(\boldsymbol{\alpha}) \geq c$ for some positive absolute constant $c$ (not depending on $n$ ). This is false, as the following example shows. Let $V_{n} \subseteq \mathbb{G}_{\mathrm{m}}^{n}$ be the hypersurface defined by the equation

$$
x_{1}+\cdots+x_{n-1}+x_{n}=0 .
$$

We claim that, as $n$ tends to $\infty$,

$$
\min _{\boldsymbol{\alpha} \in V_{n}^{*}} h(\boldsymbol{\alpha}) \rightarrow 0 .
$$

Indeed, let $n \geq 3$. Consider for instance the point $\alpha \in \mathbb{G}_{\mathrm{m}}^{n}(\overline{\mathbb{Q}})$ whose coordinates are the roots $\alpha_{1}, \ldots, \alpha_{n}$ of the polynomial $f(x)=x^{n}-2 x-6$. Observe that $f$ is 
irreducible by Eisenstein's criterion. Moreover $\alpha \in V_{n}$, because the coefficient of $x^{n-1}$ in $f$ is zero. We now show that $\boldsymbol{\alpha}$ has small height. For a non-zero integer a, let $\mathbf{a}=(\mathrm{a}, \ldots, \mathrm{a}) \in \mathbb{G}_{\mathrm{m}}^{n}$. Since $\boldsymbol{\alpha}^{n}=\mathbf{2} \cdot \boldsymbol{\alpha}+\mathbf{6}$ we obtain

$$
n h(\boldsymbol{\alpha})=h\left(\boldsymbol{\alpha}^{n}\right)=h(\mathbf{2} \cdot \boldsymbol{\alpha}+\mathbf{6}) \leq h(\mathbf{2} \cdot \boldsymbol{\alpha})+h(\mathbf{6})+\log 2 \leq h(\boldsymbol{\alpha})+\log 24 .
$$

Thus

$$
h(\boldsymbol{\alpha}) \leq \frac{\log 24}{n-1} .
$$

We claim that $\alpha \in V_{n}^{*}$. Assume on the contrary that $\alpha$ is in a torsion variety contained in $V_{n}$. From the description of [Sch], p. 163, of the torsion varieties contained in a linear variety, we see that there exist $i<j$ such that $u=\alpha_{i} / \alpha_{j}$ is a root of unity. Note that $u \neq 1$ because $f$ has distinct roots. Thus

$$
0=f\left(\alpha_{j}\right)-f\left(u \alpha_{j}\right)=\left(1-u^{n}\right) \alpha_{j}^{n}-2(1-u) \alpha_{j} .
$$

Let $\gamma=\left(1-u^{n}\right) /(1-u)$. Then $\gamma$ is an algebraic integer and $\gamma \alpha_{j}^{n-1}=2$. Passing to norms, we infer that $\pm 6=\operatorname{Norm}_{\mathbb{Q}}^{\mathbb{Q}\left(\alpha_{j}\right)}\left(\alpha_{j}\right)$ divides a power of 2. This is a contradiction. Thus $\boldsymbol{\alpha} \in V^{*}(\overline{\mathbb{Q}})$ and $h(\boldsymbol{\alpha}) \leq \frac{\log 24}{n-1}$.

\section{Geometry}

2.1. Algebraic interpolation. In the introduction, we have already mentioned the definitions of $\omega(V)$ and $\delta_{0}(V)$ for a projective variety $V \subseteq \mathbb{P}^{n}$. Let us be more precise and give some further details and useful relations.

Definition 2.1. Let $V \subseteq \mathbb{P}^{n}$ be a projective variety and let $K$ be a subfield of $\overline{\mathbb{Q}}$.

i) The obstruction index $\omega_{K}(V)$ is the minimum degree of a hypersurface defined over $K$ containing $V$.

ii) We define $\delta_{K, 0}(V)$ as the minimal degree $\delta$ such that there exists an intersection $X$ of hypersurfaces defined over $K$ of degree $\leq \delta$ such that every $\overline{\mathbb{Q}}$-irreducible component of $V$ is a $\overline{\mathbb{Q}}$-irreducible component of $X$.

iii) Suppose that $V$ is defined over $K$. We define $\delta_{K}(V)$ as the minimal degree $\delta$ such that $V$ is, as a set, the intersection of hypersurfaces defined over $K$ of degree $\leq \delta$.

If $K=\overline{\mathbb{Q}}$ we shall omit the index $\overline{\mathbb{Q}}$.

Note that the definition of $\delta_{K, 0}$ makes sense for every number field $K$, independently of the field of definition $L$ of $V$. Indeed, $V^{\prime}=\bigcup_{\sigma \in \operatorname{Gal}(\overline{\mathbb{Q}} / K)} \sigma(V)$ is defined over $K$ and the $\overline{\mathbb{Q}}$-irreducible components of $V$ are components of $V^{\prime}$. On the contrary, $\delta_{K}$ can only be defined for extensions of the field of definition of $V$. Indeed if $V$ 
is the intersection of hypersurfaces over $K$ then it is also defined over $K$. In addition, if $V$ is defined over $K$, then in the above definition ii), it is equivalent to require that every $K$-irreducible component of $V$ is a $K$-irreducible component of $X$.

Clearly, for $L$ a field extension of $K, \omega_{K} \geq \omega_{L}, \delta_{K, 0} \geq \delta_{L, 0}$ and $\delta_{K} \geq \delta_{L}$. We are now going to show that these are equalities for extensions $L$ of the field of definition $K$ of $V$.

Let $G$ be a group acting on $\mathbb{G}_{\mathrm{m}}^{n}$. For any subset $S$ of $\mathbb{G}_{\mathrm{m}}^{n}$ we define

$$
\begin{aligned}
S^{G} & =\bigcap_{g \in G} g(S), \\
G \cdot S & =\bigcup_{g \in G} g(S) .
\end{aligned}
$$

In what follows we provide relations between the obstruction indices of $V$ and $V^{G}$ in two special cases, namely for $G$ the Galois group (Lemma 2.2 below) and for $G$ the kernel of the "multiplication by $l$ " (Lemma 2.4).

Lemma 2.2. Let $K$ be a number field and let $Z$ be a hypersurface defined over some extension $L$ of $K$. Then there exist $D \leq[L: K]$ and hypersurfaces $Z_{1}, \ldots, Z_{D}$ defined over $K$ and of degree $\leq \operatorname{deg} Z$ such that

$$
Z^{\mathrm{Gal}(\overline{\mathbb{Q}} / K)}=Z_{1} \cap \cdots \cap Z_{D} .
$$

Proof. Let $F(\boldsymbol{x}) \in L[\boldsymbol{x}]$ be an equation defining $Z$. We fix a basis $\left\{e_{j}\right\}$ of $L / K$ and we write $F(\boldsymbol{x})=\sum e_{j} F_{j}(\boldsymbol{x})$ with $F_{j}(\boldsymbol{x}) \in K[\boldsymbol{x}]$. Up to order, we can suppose $F_{j}(\boldsymbol{x}) \neq 0$ for $j=1, \ldots, D$ and $F_{j}(\boldsymbol{x})=0$ for $j>D$. Define $Z_{j}$ to be the zero set of $F_{j}(\boldsymbol{x})$, for $j \leq D$. Clearly $Z^{\mathrm{Gal}(\overline{\mathbb{Q}} / K)} \supseteq Z_{1} \cap \cdots \cap Z_{D}$. We now show the reverse inclusion. Let $\boldsymbol{\alpha} \in Z^{\mathrm{Gal}(\overline{\mathbb{Q}} / K)}$. Let each $\sigma_{1}, \ldots, \sigma_{[L: K]}$ be an extension to $\overline{\mathbb{Q}}$ of each of the $[L: K]$ embeddings of $L$ in $\overline{\mathbb{Q}}$ fixing $K$. Then, for every $i$, also $\sigma_{i}^{-1}(\boldsymbol{\alpha}) \in Z^{\mathrm{Gal}(\overline{\mathbb{Q}} / K)}$. Since the $F_{j}$ are invariant under the action of any such $\sigma_{i}$, we obtain that for every $i \leq[L: K]$

$$
\begin{aligned}
0 & =\sigma_{i}\left(F\left(\sigma_{i}^{-1}(\boldsymbol{\alpha})\right)=\sigma_{i}\left(\sum e_{j} F_{j}\left(\sigma_{i}^{-1}(\boldsymbol{\alpha})\right)\right)\right. \\
& =\sigma_{i}\left(\sum e_{j}\left(\sigma_{i}^{-1} F_{j}(\boldsymbol{\alpha})\right)\right)=\sum \sigma_{i}\left(e_{j}\right) F_{j}(\boldsymbol{\alpha}) .
\end{aligned}
$$

The matrix $\left(\sigma_{i} e_{j}\right)_{i, j}$ is non singular. This implies that $F_{j}(\boldsymbol{\alpha})=0$ for all $1 \leq j \leq$ $[L: K]$. This shows the inclusion $Z^{\mathrm{Gal}(\overline{\mathbb{Q}} / K)} \subseteq Z_{1} \cap \cdots \cap Z_{D}$.

Corollary 2.3. Let $V$ be a variety defined over a number field $K$. Then $\delta_{K}(V)=$ $\delta(V), \omega_{K}(V)=\omega(V)$ and $\delta_{K, 0}(V)=\delta_{0}(V)$. 
Proof. We already mentioned that such invariants decrease by fields extensions. Then we have only to show that $\delta_{K}(V) \leq \delta(V), \omega_{K}(V) \leq \omega(V)$ and $\delta_{K, 0}(V) \leq \delta_{0}(V)$.

Let $X \supseteq V$ be an intersection of hypersurfaces of degree $\leq \delta$, for $\delta \in \mathbb{N}$. By Lemma $2.2 X^{\mathrm{Gal}(\overline{\mathbb{Q}} / K)}$ is an intersection of hypersurfaces defined over $K$, of degree $\leq \delta$. Since $V$ is defined over $K, V=V^{\mathrm{Gal}(\overline{\mathbb{Q}} / K)} \subseteq X^{\mathrm{Gal}(\overline{\mathbb{Q}} / K)}$.

Choosing $\delta=\delta(V)$ and $X=V$ we see that $\delta_{K}(V) \leq \delta(V)$. Choosing $\delta=\omega(V)$ and $X \supseteq V$ a hypersurface defined over $\overline{\mathbb{Q}}$ of minimal degree $\delta$ we see that $\omega_{K}(V) \leq$ $\omega(V)$. Choose at last $\delta=\delta_{0}(V)$ and $X \supseteq V$ such that every $\overline{\mathbb{Q}}$-irreducible component of $V$ is a $\overline{\mathbb{Q}}$-irreducible component of $X$. Let $W$ be a $\overline{\mathbb{Q}}$-irreducible component of $V$. Then $W$ is a $\overline{\mathbb{Q}}$-irreducible component of $X$. Since $V \subseteq X^{\mathrm{Gal}(\overline{\mathbb{Q}} / K)} \subseteq X$, we see that $W$ is a $\overline{\mathbb{Q}}$-irreducible component of $X^{\mathrm{Gal}(\overline{\mathbb{Q}} / K)}$, too. Thus $\delta_{K, 0}(V) \leq \delta_{0}(V)$.

We shall recall some important relations between the obstruction indices. If $V$ is equidimensional of codimension $k$, then, by a result of M. Chardin ([Cha]),

$$
\omega(V) \leq n \operatorname{deg}(V)^{1 / k} .
$$

Moreover,

$$
\omega(V) \leq \delta_{0}(V) \leq \delta(V) \leq \operatorname{deg}(V) \leq \delta_{0}(V)^{k} .
$$

The first three inequalities are immediate. The last one follows from [Phi], Corollary 5 , p. 357 (with $m=n, S=\mathbb{P}^{n}$ and $\delta=\delta_{0}(V)$ ).

2.2. An upper bound for $\delta_{0}([l] V)$. Let $V$ be an equidimensional variety and let $l \neq 0$ be an integer. We need a bound for $\delta_{0}([l] V)$. We denote by $[l]: \mathbb{G}_{\mathrm{m}}^{n} \rightarrow$ $\mathbb{G}_{\mathrm{m}}^{n}, \boldsymbol{\alpha} \mapsto \boldsymbol{\alpha}^{l}=\left(\alpha_{1}^{l}, \ldots, \alpha_{n}^{l}\right)$ the "multiplication by $l$ " and by ker $[l]$ its kernel. The following lemma is analogue to Lemma 2.2. Here we consider the action of $\operatorname{ker}[l]$, whereas in Lemma 2.2 we considered the Galois action.

Lemma 2.4. Let $Z \subset \mathbb{G}_{\mathrm{m}}^{n}$ be a hypersurface. Then, there exist $D \leq l^{n}$ and hypersurfaces $Z_{1}, \ldots, Z_{D}$ of degree $\leq \operatorname{deg} Z$ such that $\operatorname{ker}[l] \cdot Z_{j}=Z_{j}$ and

$$
Z^{\operatorname{ker}[l]}=Z_{1} \cap \cdots \cap Z_{D}
$$

Proof. Let $F(x) \in \overline{\mathbb{Q}}[\boldsymbol{x}]$ be an equation for $Z$. Performing the euclidean divisions by $l$ on the exponents of each monomial, we can write

$$
F(x)=\sum_{\lambda \in \Lambda} x^{\lambda} F_{\lambda}\left(x^{l}\right)
$$

where $\boldsymbol{x}^{l}=\left(x_{1}^{l}, \ldots, x_{n}^{l}\right)$ and $\lambda$ runs over the set $\Lambda$ of integral multi-indices $\lambda=$ $\left(\lambda_{1}, \ldots, \lambda_{n}\right)$ with $0 \leq \lambda_{i}<l$. Let $Z_{j}$ be the hypersurfaces defined by the non-trivial 
$F_{\lambda}\left(\boldsymbol{x}^{l}\right)$. Clearly $\operatorname{ker}[l] \cdot Z_{j}=Z_{j}$. Moreover $Z^{\operatorname{ker}[l]} \supseteq Z_{1} \cap \cdots \cap Z_{D}$. We now show the reverse inclusion. Let $\alpha \in Z^{\operatorname{ker}[l]}$. Then, for every $\zeta \in \operatorname{ker}[l]$,

$$
0=F(\zeta \alpha)=\sum_{\lambda \in \Lambda}(\zeta \alpha)^{\lambda} F_{\lambda}\left((\zeta \alpha)^{l}\right)=\sum_{\lambda \in \Lambda} \zeta^{\lambda} \alpha^{\lambda} F_{\lambda}\left(\alpha^{l}\right) .
$$

Let $\zeta_{i}$ varying over all elements of $\operatorname{ker}[l]$ and $\lambda_{j}$ varying over all elements of $\Lambda$. Then we can write the following homogenous linear system

$$
\left(\zeta_{i}^{\lambda_{j}}\right)_{i, j}\left(\boldsymbol{\alpha}^{\lambda_{j}} F_{\lambda_{j}}\left(\boldsymbol{\alpha}^{l}\right)\right)_{j}=\mathbf{0} .
$$

Since the matrix $\left(\zeta_{i}^{\lambda_{j}}\right)_{i, j}$ is non singular, $\left(\boldsymbol{\alpha}^{\lambda_{j}} F_{\lambda_{j}}(\boldsymbol{\alpha})\right)_{j}$ must be the zero vector. We remark that no monomial vanishes on $\mathbb{G}_{\mathrm{m}}^{n}$. Then we have $\alpha \in Z_{1} \cap \cdots \cap Z_{D}$. This shows that $Z^{\mathrm{ker}[l]} \subseteq Z_{1} \cap \cdots \cap Z_{D}$.

To estimate $\delta_{0}$, we need a generalization of Lemma 3.7 of [Amo-Via], which holds for $\overline{\mathbb{Q}}$-irreducible varieties. Here the variety is not necessarily $\overline{\mathbb{Q}}$-irreducible. In general, the lemma does not extend to all equidimensional varieties, however it extends under some additional assumptions.

Lemma 2.5. Let $V$ be a $\mathbb{Q}$-irreducible subvariety of $\mathbb{G}_{\mathrm{m}}^{n}$ and let l be a positive integer. Let $K$ be the field of definition of one of the $\overline{\mathbb{Q}}$-irreducible components of $V$. Assume that $K \cap \mathbb{Q}\left(\zeta_{l}\right)=\mathbb{Q}$, for a primitive l-th root of unity $\zeta_{l}$. Then

$$
\delta_{0}(\operatorname{ker}[l] \cdot V) \leq l^{n} \delta_{0}(V) .
$$

Proof. The first step is to prove the following remark. By definition of $\delta_{0}(V)$, there exists a variety $X$ defined by rational equations of degree $\leq \delta_{0}(V)$ such that $V$ is a $\mathbb{Q}$-irreducible component of $X$. Let $W_{1}, \ldots, W_{t}$ be the $\overline{\mathbb{Q}}$-irreducible components of $V$.

Remark 2.6. Let $\zeta \in \operatorname{ker}[l]$. Assume that for some $i$ the variety $\zeta W_{i} \subseteq X$. Then $\zeta W_{j} \subseteq X$ for any index $j$.

Proof. We remark that the Galois group permutes transitively $W_{1}, \ldots, W_{t}$. Let $K_{i}$ be the field of definition of $W_{i}$. By assumption $K_{i} \cap \mathbb{Q}(\zeta)=\mathbb{Q}$. Thus $\left[K_{i}(\zeta): K_{i}\right]=$ $[\mathbb{Q}(\zeta): \mathbb{Q}]$. Hence, for any $j=1, \ldots, t$ there exists $\tau \in \operatorname{Gal}(\overline{\mathbb{Q}} / \mathbb{Q})$ such that $\tau\left(W_{i}\right)=W_{j}$ and $\tau(\zeta)=\zeta$. We infer that $\zeta W_{j}=\tau\left(\zeta W_{i}\right)$ is included in $\tau(X)=X$.

In what follows we say that a $\overline{\mathbb{Q}}$-irreducible variety $W \subseteq \mathbb{G}_{\mathrm{m}}^{n}$ is imbedded in a variety $X \subseteq \mathbb{G}_{\mathrm{m}}^{n}$ if $V$ is a subset of $X$ but not an irreducible component of $X$. Let 
us denote $W=W_{1}$. Let $S$ be the set of $\zeta \in \operatorname{ker}[l]$ such that $\zeta W$ is imbedded in $X$. Then, by the remark above, $V \subseteq \zeta^{-1} X$. We define

$$
X^{\prime}=X \cap \bigcap_{\zeta \in S} \zeta^{-1} X
$$

Note that $V \subseteq X^{\prime}$. Furthermore, the varieties $X$ and $\zeta^{-1} X$ are intersections of hypersurfaces of degree $\leq \delta_{0}(V)$. Thus $\delta\left(X^{\prime}\right) \leq \delta_{0}(V)$.

We shall show that no translate $\zeta W_{j}$ is imbedded in $X^{\prime}$. Assume by contradiction that $\zeta W_{j}$ was imbedded in $X^{\prime}$ for some $\zeta \in \operatorname{ker}[l]$ and for some $j \in\{1, \ldots, n\}$. We will prove that $1 \in S$. Then $W$ would be imbedded in $X$, which contradicts the fact that $W$ is a component of $X$. Since $\zeta$ has finite order, to prove $\mathbf{1} \in S$ it is sufficient to prove that $\zeta^{n} \in S$, for all positive integers $n$. We proceed by induction. Since $X^{\prime} \subseteq X, \zeta W_{j}$ is imbedded in $X$ and so $\zeta \in S$. We now assume $\zeta^{n} \in S$ for some $n \geq 1$ and we prove that $\zeta^{n+1} \in S$. Since $X^{\prime} \subseteq \zeta^{-n} X, \zeta W_{j}$ is imbedded in $\zeta^{-n} X$. Thus $\zeta^{n+1} W_{j}$ is imbedded in $X$ and $\zeta^{n+1} \in S$.

We now define

$$
Y=\operatorname{ker}[l] \cdot X^{\prime} .
$$

Clearly $\operatorname{ker}[l] \cdot V \subseteq Y$ and $\delta(Y) \leq l^{n} \delta\left(X^{\prime}\right) \leq l^{n} \delta_{0}(V)$. Let $\zeta W_{j}(\zeta \in \operatorname{ker}[l]$, $j \in\{1, \ldots, t\}$ ) be a $\overline{\mathbb{Q}}$-irreducible component of $\operatorname{ker}[l] \cdot V$. Assume by contradiction $\zeta W_{j}$ imbedded in $Y$. Then $\zeta W_{j}$ is imbedded in $\eta X^{\prime}$ for some $\eta \in \operatorname{ker}[l]$. Thus $\eta^{-1} \zeta W_{j}$ is imbedded in $X^{\prime}$, which contradicts the construction of $X^{\prime}$.

At last we provide the necessary upper bound for $\delta_{0}([l] V)$.

Proposition 2.7. Let $V$ be a $\mathbb{Q}$-irreducible subvariety of $\mathbb{G}_{\mathrm{m}}^{n}$ and let l be a positive integer. Let $K$ be the field of definition of one of the $\overline{\mathbb{Q}}$-irreducible component of $V$. Assume that $K \cap \mathbb{Q}\left(\zeta_{l}\right)=\mathbb{Q}$. Then

$$
\delta_{0}([l] V) \leq l^{n-1} \delta_{0}(V) .
$$

Proof. By Lemma 2.5 there exist hypersurfaces $Z_{1}, \ldots, Z_{r}$ of degree $\leq l^{n} \delta_{0}(V)$ such that every $\overline{\mathbb{Q}}$-irreducible component of $\operatorname{ker}[l] \cdot V$ is a component of $Z_{1} \cap \cdots \cap Z_{r}$. By Lemma 2.4 we can assume $\operatorname{ker}[l] \cdot Z_{i}=Z_{i}$. Thus

$$
[l] V \subseteq[l] Z_{1} \cap \cdots \cap[l] Z_{r}
$$

and $\operatorname{deg}\left([l] Z_{i}\right)=l^{-1} \operatorname{deg}\left(Z_{i}\right)$. We now show that each component of $[l] V$ is isolated in such an intersection. Suppose on the contrary that $U$ is a $\overline{\mathbb{Q}}$-irreducible component of $V$ such that

$$
[l] U \subsetneq Y \subseteq[l] Z_{1} \cap \cdots \cap[l] Z_{r}
$$

for some $\overline{\mathbb{Q}}$-irreducible $Y$. Then there exists a $\overline{\mathbb{Q}}$-irreducible component $Y^{\prime}$ of $[l]^{-1} Y$ such that

$$
U \subsetneq Y^{\prime} \subseteq\left(\operatorname{ker}[l] \cdot Z_{1}\right) \cap \cdots \cap\left(\operatorname{ker}[l] \cdot Z_{r}\right)=Z_{1} \cap \cdots \cap Z_{r} .
$$


This contradicts the fact that each component of $V$ is isolated in $Z_{1} \cap \cdots \cap Z_{r}$.

2.3. Exceptional primes. Let $V \subseteq \mathbb{G}_{\mathrm{m}}^{n}$ be a $\mathbb{Q}$-irreducible variety and let $\wp$ be a finite set of primes. In what follows, we need a lower bound for the degree of $\bigcup_{p \in \wp}[p] V$ and an upper bound for $\delta_{0}([p] V)$ for $p \in \wp$. This holds outside a set of "bad" primes. One has to ensure that there are few bad primes. This is the object of the next proposition. Part of the proof was already in [Amo-Dav], Section 2. We prefer to reproduce the integral argument.

Proposition 2.8. Let $V \subseteq \mathbb{G}_{\mathrm{m}}^{n}$ be a $\mathbb{Q}$-irreducible variety of dimension $d$. Assume that $V$ is not a union of torsion varieties. Then there exists a set of prime numbers $E(V)$ of cardinality

$$
|E(V)| \leq \frac{d+1}{\log 2} \log \operatorname{deg}(V)
$$

such that for all prime numbers $p \notin E(V)$,

$$
\delta_{0}([p] V) \leq p^{n-1} \delta_{0}(V)
$$

and, for all finite subsets $\wp$ of primes lying outside $E(V)$,

$$
\operatorname{deg}\left(\bigcup_{p \in \wp}[p] V\right) \geq|\wp| \operatorname{deg}(V) .
$$

Proof. We remark that the Galois group permutes transitively the $\overline{\mathbb{Q}}$-irreducible components $W=W_{1}, \ldots, W_{k}$ of $V$. We recall the definition of stabilizer:

$$
\operatorname{Stab}(W)=\left\{\alpha \in \mathbb{G}_{\mathrm{m}}^{n} \text { such that } \alpha W=W\right\} .
$$

Define $H=\operatorname{Stab}(W) / \operatorname{Stab}(W)^{0}$ where $\operatorname{Stab}(W)^{0}$ is the connected component of $\operatorname{Stab}(W)$ through the neutral element. Then, $H$ is a finite group of cardinality

$$
|H| \leq \operatorname{deg}(\operatorname{Stab} W) \leq \operatorname{deg}(W)^{d+1} .
$$

We denote $d_{0}=\operatorname{dim} \operatorname{Stab}(W) \leq d$. We remark that for any natural number $l$, it holds that

$$
|\operatorname{ker}[l] \cap \operatorname{Stab}(W)|=\left|\operatorname{ker}[l] \cap \operatorname{Stab}(W)^{0}\right| \cdot|\operatorname{ker}[l] \cap H|=l^{d_{0}}|\operatorname{ker}[l] \cap H|,
$$

where we identify $[l]$ with the "multiplication" by $l$ in the quotient $\mathbb{G}_{\mathrm{m}}^{n} / \operatorname{Stab}(W)^{0}$. Furthermore, denote by $K$ the field of definition of $W$. Then $[K: \mathbb{Q}]=k$.

Let $E_{1}$ be the set of prime numbers $p$ such that $p$ divides $|H|$. Let $E_{2}$ be the set of primes $p$ such that $[p] W=[p] W_{i}$ for some $1<i \leq k$. Let $E_{3}$ be the set of primes $p$ such that $K \cap \mathbb{Q}\left(\zeta_{p}\right) \neq \mathbb{Q}$, where as usual $\zeta_{p}$ is a primitive $p$-th root of unity. We define

$$
E(V)=E_{1} \cup E_{2} \cup E_{3}
$$


Since $E_{3} \subseteq E(V)$, Proposition 2.7 shows that the upper bound (2.6) holds.

We now prove (2.7). First we show that

$$
p \nmid|H| \Longrightarrow \operatorname{deg}\left([p] W_{i}\right) \geq \operatorname{deg}\left(W_{i}\right), \quad \text { for } i=1, \ldots, k .
$$

If $p \nmid|H|$, then $|\operatorname{ker}[p] \cap H|=1$. By the degree formula for the image of the multiplication by $p$ (see for instance [Dav-Phi], Proposition 2.1 (i)),

$$
\operatorname{deg}([p] W)=p^{d-d_{0}}|\operatorname{ker}[p] \cap H|^{-1} \operatorname{deg}(W)=p^{d-d_{0}} \operatorname{deg}(W) \geq \operatorname{deg}(W) .
$$

This shows (2.9).

We now show that, for $l_{1}, l_{2}$ natural integers,

$$
\begin{aligned}
& V \neq \text { union of torsion varieties and } l_{1} \neq l_{2} \\
& \quad \Longrightarrow\left[l_{1}\right] W_{i} \neq\left[l_{2}\right] W_{j} \text { for } i, j=1, \ldots, k .
\end{aligned}
$$

Assume on the contrary that $\left[l_{1}\right] W$ is a Galois conjugate to $\left[l_{2}\right] W$. Since the multiplication by natural numbers commute with the Galois action, the same holds replacing $l_{i}$ by $l_{i}^{r}$ for $r \in \mathbb{N}$, as well. We can suppose $l_{1}<l_{2}$. Let $\hat{h}$ be the normalised height for subvarieties of $\mathbb{G}_{\mathrm{m}}^{n}$ (see for instance [Dav-Phi]). Then $\hat{h}\left(\left[l_{1}\right] W\right)=\hat{h}\left(\left[l_{2}\right] W\right)$. By the height formula for the image of the multiplication by an integer ([Dav-Phi], Proposition 2.1 (i)), we obtain

$$
\begin{aligned}
l_{1}^{d-d_{0}+1}\left|\operatorname{ker}\left[l_{1}\right] \cap H\right|^{-1} \hat{h}(W) & =\hat{h}\left(\left[l_{1}\right] W\right)=\hat{h}\left(\left[l_{2}\right] W\right) \\
& =l_{2}^{d-d_{0}+1}\left|\operatorname{ker}\left[l_{2}\right] \cap H\right|^{-1} \hat{h}(W) .
\end{aligned}
$$

Since $V$ is not a union of torsion varieties, $W$ is not torsion. Then $\hat{h}(W)>0$. Thus

$$
l_{2} / l_{1} \leq\left(l_{2} / l_{1}\right)^{d-d_{0}+1} \leq \frac{\left|\operatorname{ker}\left[l_{2}\right] \cap H\right|}{\left|\operatorname{ker}\left[l_{1}\right] \cap H\right|} \leq|H| .
$$

Replacing $l_{1}$ and $l_{2}$ with $l_{1}^{r}$ and $l_{2}^{r}$ and letting $r \rightarrow+\infty$ we get a contradiction.

Let $\wp$ be a set of primes lying outside $E(V)$ and assume that $V$ is not a union of torsion varieties. The statements (2.9) and (2.10) and the definition of $E(V)$ show that

$$
\begin{aligned}
\operatorname{deg}\left(\bigcup_{p \in \wp}[p] V\right) & =\operatorname{deg}\left(\bigcup_{j=1}^{k} \bigcup_{p \in \wp}[p] W_{j}\right)=\sum_{j=1}^{k} \sum_{p \in \wp} \operatorname{deg}\left([p] W_{j}\right) \\
& \geq \sum_{j=1}^{k} \sum_{p \in \wp} \operatorname{deg}\left(W_{j}\right)=|\wp| \operatorname{deg}(V) .
\end{aligned}
$$


To conclude the proof, we need to provide an upper bound for the cardinality of $E(V)=E_{1} \cup E_{2} \cup E_{3}$. First we remark that by (2.8) the set $E_{1}$ of primes $p$ dividing $|H|$ has cardinality

$$
\leq \frac{\log |H|}{\log 2} \leq \frac{d+1}{\log 2} \log \operatorname{deg}(W)=\frac{d+1}{\log 2} \log (\operatorname{deg}(V) / k) .
$$

Below we detail the proof that the set $E_{2}$ has cardinality

$$
\left|E_{2}\right| \leq \frac{\log k}{\log 2} .
$$

We have still to estimate the cardinality of the set $E_{3}$ of primes $p$ such that $K \cap$ $\mathbb{Q}\left(\zeta_{p}\right) \neq \mathbb{Q}$. It holds that

$$
\left|E_{3}\right| \leq \frac{\log k}{\log 2} .
$$

Indeed, for $l \in \mathbb{N}$, define $K_{l}=K \cap \mathbb{Q}\left(\zeta_{l}\right)$. Thus, $K_{l} / \mathbb{Q}$ is Galois. We note that for $n, m \in \mathbb{N}$ coprime, $K_{n} \cap K_{m}=\mathbb{Q}$ and $K_{n} K_{m} \subseteq K_{n m}$. By induction we easily see that

$$
k=[K: \mathbb{Q}] \geq\left[\prod_{p \in E_{3}} K_{p}: \mathbb{Q}\right]=\prod_{p \in E_{3}}\left[K_{p}: \mathbb{Q}\right] \geq 2^{\left|E_{3}\right|} .
$$

This is equivalent to (2.12). We conclude that

$$
\begin{aligned}
|E(V)| \leq\left|E_{1}\right|+\left|E_{2}\right|+\left|E_{3}\right| & \leq \frac{d+1}{\log 2} \log (\operatorname{deg}(V) / k)+\frac{2 \log k}{\log 2} \\
& \leq \frac{d+1}{\log 2} \log (\operatorname{deg}(V))+\frac{1-d}{\log 2} \log k \\
& \leq \frac{d+1}{\log 2} \log \operatorname{deg}(V)
\end{aligned}
$$

as required.

The upper bound for $\left|E_{2}\right|$ is a variant of the corresponding lemma of Dobrowolski ([Dob], Lemma 3). For a natural integer $l$ and for $i \in\{1, \ldots, k\}$, let

$$
\mathcal{I}(l, i)=\left\{j,[l] W_{i}=[l] W_{j}\right\} .
$$

Thus, for a fixed $l$, these sets have the same cardinality. Moreover, $p \in E_{2}$ if and only if $\mathcal{I}(p, 1) \geq 2$.

Let $l_{1}, l_{2}$ be coprime integers. Then, by the definition of the sets $\mathcal{I}$,

$$
\mathcal{I}\left(l_{1} l_{2}, i\right) \supseteq \bigcup_{j \in \mathcal{I}\left(l_{1}, i\right)} \mathfrak{I}\left(l_{2}, j\right) .
$$

Indeed, if $m \in \mathcal{I}\left(l_{2}, j\right)$ for some $j \in \mathcal{I}\left(l_{1}, i\right)$, we have $\left[l_{2}\right] W_{j}=\left[l_{2}\right] W_{m}$ and $\left[l_{1}\right] W_{i}=\left[l_{1}\right] W_{j}$ which implies $\left[l_{1} l_{2}\right] W_{i}=\left[l_{1} l_{2}\right] W_{j}=\left[l_{1} l_{2}\right] W_{m}$. This immediately 
gives the inclusion. Moreover, for $j \in \mathcal{I}\left(l_{1}, i\right)$ the sets $\mathcal{I}\left(l_{2}, j\right)$ are pairwise distinct. Indeed, let $j_{1}, j_{2} \in \mathcal{I}\left(l_{1}, i\right)$ such that $\mathcal{I}\left(l_{2}, j_{1}\right) \cap \mathcal{I}\left(l_{2}, j_{2}\right) \neq \emptyset$. Then $\left[l_{1}\right] W_{j_{1}}=$ $\left[l_{1}\right] W_{j_{2}}$ and $\left[l_{2}\right] W_{j_{1}}=\left[l_{2}\right] W_{j_{2}}$. Thus, there exist $\boldsymbol{x}_{1} \in \operatorname{ker}\left[l_{1}\right]$ and $\boldsymbol{x}_{2} \in \operatorname{ker}\left[l_{2}\right]$ such that $W_{j_{2}}=\boldsymbol{x}_{1} W_{j_{1}}=\boldsymbol{x}_{2} W_{j_{1}}$. This implies that $\boldsymbol{x}_{2}^{-1} \boldsymbol{x}_{1} \in \operatorname{Stab}\left(W_{j_{1}}\right)$. Since $l_{1}, l_{2}$ are coprime, by the Bézout identity, there exist integers $u_{1}, u_{2}$ such that $u_{1} l_{1}+u_{2} l_{2}=1$. Thus

$$
\boldsymbol{x}_{1}=\boldsymbol{x}_{1}^{1-u_{1} l_{1}}=\boldsymbol{x}_{1}^{u_{2} l_{2}}=\left(\boldsymbol{x}_{2}^{-1} \boldsymbol{x}_{1}\right)^{u_{2} l_{2}} \in \operatorname{Stab}\left(W_{j_{1}}\right) .
$$

Hence $W_{j_{2}}=\boldsymbol{x}_{1} W_{j_{1}}=W_{j_{1}}$, and $j_{1}=j_{2}$. This proves that (2.13) is a disjoint union. We infer

$$
\left|\mathcal{I}\left(l_{1} l_{2}, i\right)\right| \geq \sum_{j \in \mathcal{I}\left(l_{1}, i\right)}\left|\mathcal{I}\left(l_{2}, j\right)\right|=\left|\mathcal{I}\left(l_{1}, 1\right)\right|\left|\mathcal{I}\left(l_{2}, 1\right)\right| .
$$

Iterating this process, we see that

$$
k \geq\left|\mathcal{I}\left(\prod_{p \in E_{2}} p, 1\right)\right| \geq \prod_{p \in E_{2}}|\mathcal{I}(p, 1)| \geq 2^{\left|E_{2}\right|},
$$

which proves (2.11) and concludes the proof of the proposition.

We remark that the inequalities (2.9) and (2.11) in the proof of the previous proposition hold even for a $\mathbb{Q}$-irreducible variety which is the union of torsion varieties.

\section{Diophantine analysis}

3.1. Coding the information. Let $I \subset \overline{\mathbb{Q}}[\boldsymbol{x}]$ be a homogeneous radical ideal, where $\boldsymbol{x}=\left(x_{1}, \ldots, x_{n}\right)$. For $v \in \mathbb{N}$ we denote by $H(\overline{\mathbb{Q}}[\boldsymbol{x}] / I ; v)$ the Hilbert function $\operatorname{dim}[\overline{\mathbb{Q}}[\boldsymbol{x}] / I]_{v}$. Let $T$ be a positive integer. We denote by $I^{(T)}$ the $T$-symbolic power of $I$, i.e. the ideal of polynomials vanishing on the variety defined by $I$ with multiplicity at least $T$. Let $V$ be a variety of $\mathbb{G}_{\mathrm{m}}^{n}$. Let $I$ be the radical homogeneous ideal in $\overline{\mathbb{Q}}[\boldsymbol{x}]$ defining the Zariski closure of $V$ in $\mathbb{P}^{n}$. By abuse of notation, we set $H(V ; v)=H(\overline{\mathbb{Q}}[\boldsymbol{x}] / I ; v)$ and $H(V, T ; v)=H\left(\overline{\mathbb{Q}}[\boldsymbol{x}] / I^{(T)} ; v\right)$.

Proposition 3.1. Let $v, T$ be positive integers and let $\wp$ be a finite set of prime numbers. Let $V$ be a subvariety of $\mathbb{G}_{\mathrm{m}}^{n}$ defined over $\mathbb{Q}$. Define $V^{\prime}=\bigcup[p] V$ for $p$ running over $\wp$. Then, for some $p \in \wp$,

$$
\hat{\mu}^{\mathrm{ess}}(V) \geq \frac{1}{p v}\left(T \log p-\frac{T H(V, T ; v)}{H\left(V^{\prime} ; v\right)}(\log (v+1)+\log p)-n \log (v+1)\right) .
$$

Proof. Denote for simplicity $H=H(V, T ; v)$ and $H^{\prime}=H\left(V^{\prime} ; v\right)$ and choose a real $\varepsilon$ such that $\varepsilon>\hat{\mu}^{\text {ess }}(V)$. We remark that the lower bound for $\hat{\mu}^{\text {ess }}(V)$ of the proposition is obviously negative if $H \geq H^{\prime}$. Hence we assume $H^{\prime}>H$. 
As usual in diophantine approximation, we first construct the auxiliary function. We are going to show that there exists an homogeneous polynomial $F \in \mathbb{Q}[\boldsymbol{x}]_{\mathcal{V}}$ vanishing on $V$ with multiplicity $\geq T$ but not vanishing identically on $V^{\prime}$ and such that the Weil height of the vector of its coefficients satisfies

$$
\left(H^{\prime}-H\right) h(F) \leq H((T+n) \log (v+1)+v \varepsilon) .
$$

Consider the vector space $E$ of homogeneous polynomials $F \in \mathbb{Q}[\boldsymbol{x}]_{v}$ vanishing on $V$ with multiplicity $\geq T$. Let

$$
L=\left(\begin{array}{c}
v+n \\
n
\end{array}\right)
$$

Then $\operatorname{dim}(E)=L-H$. Note that $L-H>L-H^{\prime} \geq 0$. Thus $\operatorname{dim}(E) \geq 1$. Then there exists a basis $F_{1}, \ldots, F_{L-H}$ of $E$ such that

$$
\sum_{j=1}^{L-H} h\left(F_{j}\right) \leq H((T+n) \log (v+1)+v \varepsilon) .
$$

This is a standard application of Bombieri and Vaaler's version of Siegel's lemma. The proof can be found in [Amo-Dav], Theorem 4.1. We briefly give a sketch. Theorem 8 of [Bom-Vaa] shows that there exists a basis $\left\{F_{1}, \ldots, F_{L-H}\right\}$ of $E$ such that $\sum_{j=1}^{L-H} h\left(F_{j}\right)$ is bounded by the logarithmic $L_{2}$-height (defined choosing the $L_{2}$ norm at the infinite places) $h_{2}(E)$. By the duality principle (see the proof of Theorem 9 of [Bom-Vaa]) $h_{2}(E)$ is equal to the $L_{2}$-height of the vector space $E^{\perp}$ of dimension $H$. Given $\boldsymbol{\alpha}=\left(\alpha_{1}, \ldots, \alpha_{n}\right) \in \mathbb{G}_{\mathrm{m}}^{n}(\overline{\mathbb{Q}})$ and a multi-index $\lambda=\left(\lambda_{1}, \ldots, \lambda_{n}\right) \in \mathbb{N}^{n}$ we define $\boldsymbol{\alpha}^{\boldsymbol{\lambda}}=\alpha_{1}^{\lambda_{1}} \ldots \alpha_{n}^{\lambda_{n}}$. Given two multi-indices $\boldsymbol{\lambda}, \boldsymbol{\mu}$ we write $\left(\begin{array}{l}\lambda \\ \boldsymbol{\mu}\end{array}\right)$ for the product over $j$ of $\left(\begin{array}{l}\lambda_{j} \\ \mu_{j}\end{array}\right)$. Since $V(\varepsilon)$ is Zariski-dense in $V$, the space $E^{\perp}$ is spanned by the vectors

$$
\left(\left(\begin{array}{l}
\lambda \\
\mu
\end{array}\right) \boldsymbol{\alpha}^{\lambda-\mu}\right)_{|\lambda| \leq \nu} \quad(\alpha \in V(\varepsilon),|\mu| \leq T)
$$

of $L_{2}$-height $\leq(T+n) \log (v+1)+v \varepsilon$ (use $\left.\sum_{|\lambda| \leq v}\left(\begin{array}{l}\lambda \\ \mu\end{array}\right) \leq(v+1)^{T+n}\right)$. Since the $L_{2}$-height of a vector space is bounded by the sum of the $L_{2}$-height of a basis (by an application of Hadamard's inequality, [Bom-Vaa], equation (2.6)) we find that $h_{2}(E) \leq H((T+n) \log (v+1)+v \varepsilon)$. Then equation (3.15) is proved.

We can assume $F_{1}, \ldots, F_{L-H} \in \mathbb{Z}[\boldsymbol{x}]$ and $h\left(F_{1}\right) \leq \cdots \leq h\left(F_{L-H}\right)$. We claim that there exists $j_{0} \leq L-H^{\prime}+1$ such that $F_{j_{0}}$ does not vanish on $V^{\prime}$. Indeed, if all $F_{1}, \ldots, F_{L-H^{\prime}+1}$ vanish on $V^{\prime}$, then $H^{\prime} \leq L-\left(L-H^{\prime}+1\right)=H^{\prime}-1$. Let $F=F_{j_{0}}$. Then

$$
\sum_{j=1}^{L-H} h\left(F_{j}\right) \geq\left(L-H-j_{0}+1\right) h(F) \geq\left(H^{\prime}-H\right) h(F) .
$$


Using (3.15) we deduce that $h(F)$ satisfy (3.14).

The extrapolation step is based on a generalization of Dobrowolski's main lemma ([Dob], lemme 1). We recall that $F$ does not vanish on $V^{\prime}$ and $\varepsilon>\hat{\mu}^{\text {ess }}(V)$. Then there exists $\alpha \in V(\varepsilon)$ such that $F\left(\boldsymbol{\alpha}^{p}\right) \neq 0$ for some prime $p \in \wp$. Let $v$ be a place dividing $p$. By [Amo-Dav], Theorem 3.1,

$$
\left|F\left(\boldsymbol{\alpha}^{p}\right)\right|_{v} \leq p^{-T}|\boldsymbol{\alpha}|_{v}^{p v}
$$

where $|\boldsymbol{\alpha}|=\max \left\{1,\left|\alpha_{1}\right|_{v}, \ldots,\left|\alpha_{n}\right|_{v}\right\}$. Moreover, for an arbitrary place $v$,

$$
\left|F\left(\boldsymbol{\alpha}^{p}\right)\right|_{v} \leq \begin{cases}|\boldsymbol{\alpha}|_{v}^{p v} & \text { if } v \nmid \infty \\ L|F|_{v}|\boldsymbol{\alpha}|_{v}^{p v} & \text { if } v \mid \infty\end{cases}
$$

Note that $L \leq(v+1)^{n}$ and $h(\boldsymbol{\alpha}) \leq \varepsilon$. The product formula gives

$$
0 \leq-T \log p+n \log (v+1)+h(F)+p v \varepsilon .
$$

Comparing with (3.14) we get

$$
\begin{aligned}
\left(H^{\prime}-H\right)(T \log p-n \log (v+1)-p \nu \varepsilon) & \leq H((T+n) \log (v+1)+v \varepsilon) \\
& \leq H((T+n) \log (v+1)+p v \varepsilon),
\end{aligned}
$$

which easily implies our claim.

3.2. Decoding the information. To decode the information of Proposition 3.1 we need an upper bound for the Hilbert function. The proposition below follows from a result of M. Chardin [Cha]. It is proved in Lemma 2.5 of [Amo-Dav].

Proposition 3.2. Let $V \subseteq \mathbb{P}_{n}$ be an equidimensional variety of dimension $d$ and codimension $k=n-d$. Let $v, T$ be positive integers. Then

$$
H(V, T ; v) \leq\left(\begin{array}{c}
T-1+k \\
k
\end{array}\right)\left(\begin{array}{c}
v+d \\
d
\end{array}\right) \operatorname{deg}(V) .
$$

We also need a sharp lower bound for the Hilbert function. This is a deep result of M. Chardin and P. Philippon. Let $K$ be a subfield of $\overline{\mathbb{Q}}$ and let $V$ be a $K$-irreducible variety. They prove ([Cha-Phi], Corollary 3 ) that for an equidimensional $V$,

$$
H(V ; v) \geq\left(\begin{array}{c}
v+d-m \\
d
\end{array}\right) \operatorname{deg}(V)
$$

for $v>m$ and $m=k\left(\delta_{0}(V)-1\right)$.

We need a generalization of this result. Consider finitely many equidimensional varieties $V_{j}$ of the same dimension $d$. Let $k=n-d$,

$$
m=-1+\sum_{j}\left(k\left(\delta_{0}\left(V_{j}\right)-1\right)+1\right)<k \sum_{j} \delta_{0}\left(V_{j}\right) .
$$


Let us consider the equidimensional variety $V^{\prime}=\bigcup V_{j}$. In the appendix of this article, M. Chardin and P. Philippon prove (see Subsection 6.1)

$$
H\left(V^{\prime} ; v\right) \geq\left(\begin{array}{c}
v+d-m \\
d
\end{array}\right) \operatorname{deg}\left(V^{\prime}\right)
$$

for $v>m$.

Let $\wp$ be a set of prime numbers. We apply the previous result to $V^{\prime}=\bigcup_{p \in \wp}[p] V$. Using the upper bound (2.6) of Proposition 2.8 and (3.17) we get:

Proposition 3.3. Let $V \subseteq \mathbb{G}_{\mathrm{m}}^{n}$ be a $\mathbb{Q}$-irreducible variety of dimension d and codimension $k=n-d$ which is not a union of torsion varieties. Let $N$ be a positive real number and let $\wp$ be a set of prime numbers with $p \leq N$ lying outside the set $E(V)$ of Proposition 2.8. Define

$$
V^{\prime}=\bigcup_{p \in \wp}[p] V
$$

and

$$
m=\left[k N^{n} \delta_{0}(V)\right] .
$$

Then for any $v \geq m$ we have

$$
H\left(V^{\prime} ; v\right) \geq\left(\begin{array}{c}
v+d-m \\
d
\end{array}\right) \operatorname{deg}\left(V^{\prime}\right)
$$

We are now ready to prove the main result of this section, Theorem 1.2. Let us recall the statement.

Theorem 1.2. Let $V$ be a variety of $\mathbb{G}_{\mathrm{m}}^{n}$ of codimension $k$, defined and irreducible over $\mathbb{Q}$. Assume that $V$ is not a union of torsion varieties. Let

$$
\theta_{0}=\delta_{0}(V)\left(52 n^{2} \log \left(n^{2} \delta_{0}(V)\right)\right)^{(n+1)(k+1)} .
$$

Then there exists a hypersurface $Z$ defined over $\mathbb{Q}$ of degree at most $\theta_{0}$ which does not contain $V$ and such that

$$
V\left(\theta_{0}^{-1}\right) \subseteq V \cap Z
$$

Proof. For simplicity, denote $\delta_{0}=\delta_{0}(V)$. We prove a slightly more precise result. Namely that

$$
V\left(\delta_{0}^{-1} n^{-2}\left(39 n^{2} \log \left(n^{2} \delta_{0}\right)\right)^{-(n+1)(k+1)+1}\right)
$$

is contained in a hypersurface $Z$ defined over $\mathbb{Q}$, such that $V \nsubseteq Z$ and

$$
\operatorname{deg} Z \leq \delta_{0} n^{2}\left(39 n^{2} \log \left(n^{2} \delta_{0}\right)\right)^{(n+1)(k+1)} .
$$


Since $39 n^{2 /((n+1)(k+1)} \leq 39 n^{1 /(n+1)} \leq 39 \cdot 4^{1 / 5} \leq 52$ this statement implies the statement of Theorem 1.2. Let

$$
N=\left(39 n^{2} \log \left(n^{2} \delta_{0}\right)\right)^{k+1} .
$$

We need a lower and an upper bound for $\log N$. We have

$$
\log N \geq 2 \log (39 \cdot 4 \log 4) \geq 10.75
$$

and (using $\log x<\sqrt{x}$ for $x>0, k+1 \leq 1.5 n$ and $39 \leq 2^{5.29} \leq n^{5.29}$ )

$$
\begin{aligned}
\log N & \leq(k+1) \log \left(39 n^{2} \cdot \sqrt{n^{2} \delta_{0}}\right) \\
& \leq 1.5 n \log \left(n^{8.29} \delta_{0}\right) \leq 6.22 n \log \left(n^{2} \delta_{0}\right) .
\end{aligned}
$$

We define $\wp$ as the set of prime numbers $p$ such that $N^{3 / 4} \leq p \leq N$ and $p \notin E(V)$ where $E(V)$ is as in Proposition 2.8. Thus

$$
|\wp| \geq \pi(N)-\pi\left(N^{3 / 4}\right)-|E(V)|,
$$

where, as usual, $\pi(t)$ is the cardinality of the set of prime numbers $\leq t$. By Theorem 1 of [Ros-Sch] we have, for $t \geq 59$,

$$
\frac{t}{\log t}+\frac{t}{2(\log t)^{2}}<\pi(t) \leq \frac{t}{\log t}+\frac{3 t}{2(\log t)^{2}} .
$$

By Proposition 2.8 and by the last inequality in (2.5),

$$
\begin{aligned}
|E(V)| / \sqrt{N} & \leq \frac{d+1}{\log 2} \log \operatorname{deg}(V) \cdot \frac{1}{\left(39 n^{2} \log \left(n^{2} \delta_{0}\right)\right)^{(k+1) / 2}} \\
& \leq \frac{n k \log \delta_{0}}{\log 2 \cdot 39 n^{2} \log \left(n^{2} \delta_{0}\right)} \leq \frac{1}{39 \log 2} .
\end{aligned}
$$

Thus $|\wp| \geq \frac{f(N) N}{\log N}$, where

$$
f(t)=1+\frac{1}{2 \log t}-\frac{1}{t^{1 / 4} \cdot 3 / 4}-\frac{3}{2 t^{1 / 4}(3 / 4)^{2} \log t}-\frac{\log t}{39(\log 2) t^{1 / 2}} .
$$

Since $f(t) \geq 0.937$ for $\log t \geq 10.75$, we obtain,

$$
|\wp| \geq \frac{0.937 N}{\log N}
$$

As in Proposition 3.1, we set

$$
V^{\prime}=\bigcup_{p \in \wp}[p] V
$$


We constructed $\wp$ such that $\wp \cap E(V)=\emptyset$. Then, by Proposition 2.8,

$$
\operatorname{deg}\left(V^{\prime}\right) \geq|\wp| \operatorname{deg}(V) \geq \frac{0.937 N}{\log N} \operatorname{deg}(V) .
$$

As in the statement of Proposition 3.3, let $m=\left[k N^{n} \delta_{0}\right]$. Choose

$$
v=m d+m \quad \text { and } \quad T=\left[39 n^{2} \log \left(n^{2} \delta_{0}\right)\right] .
$$

We remark that

$$
v+1 \leq n^{2} N^{n} \delta_{0}
$$

Let

$$
\theta:=\delta_{0} n^{2}\left(39 n^{2} \log \left(n^{2} \delta_{0}\right)\right)^{(n+1)(k+1)-1} .
$$

Let $W$ be the Zariski closure of the set $V\left(\theta^{-1}\right)$ and let $W^{\prime}=\bigcup_{p \in \wp}[p] W$. We remark that $W$ is defined over $\mathbb{Q}$ because the small points of $V$ are invariant under the Galois action. Then

$$
\hat{\mu}^{\mathrm{ess}}(W) \leq \theta^{-1} .
$$

Furthermore

$$
H(W, T ; v) \leq H(V, T ; v) \text { and } H\left(W^{\prime} ; \nu\right) \leq H\left(V^{\prime} ; \nu\right) .
$$

We are going to prove that the last inequality is strict. Assume on the contrary that

$$
H\left(W^{\prime} ; v\right)=H\left(V^{\prime} ; v\right) .
$$

Apply Proposition 3.2 to $V$ and Proposition 3.3 to $V^{\prime}$. Then, by (3.20),

$$
\frac{H(W, T ; v)}{H\left(W^{\prime} ; v\right)} \leq \frac{H(V, T ; v)}{H\left(V^{\prime} ; v\right)} \leq \frac{\left(\begin{array}{c}
T-1+k \\
k
\end{array}\right)\left(\begin{array}{c}
v+d \\
d
\end{array}\right)}{\left(\begin{array}{c}
v+d-m \\
d
\end{array}\right)} \times \frac{\log N}{0.937 N} .
$$

We remark that $\left(\begin{array}{c}T-1+k \\ k\end{array}\right) \leq T^{k}$. Moreover, by the choice $v=m d+m$,

$$
\left(\begin{array}{c}
v+d \\
d
\end{array}\right)\left(\begin{array}{c}
v+d-m \\
d
\end{array}\right)^{-1}=\prod_{j=1}^{d} \frac{v+j}{v-m+j} \leq\left(1+\frac{m}{v-m}\right)^{d}=\left(1+\frac{1}{d}\right)^{d} \leq e .
$$

Thus,

$$
\lambda:=\frac{T H(W, T ; v)}{H\left(W^{\prime} ; v\right)} \leq \frac{e(\log N) T^{k+1}}{0.937 N} \leq 2.91 \log N .
$$

By Proposition 3.1 (with $V$ replaced by $W$ ) there exists a prime $p \in \wp$ such that $\theta^{-1} \geq \frac{1}{p v}((T+1) \log p-\lambda(\log (v+1)+\log N)-n \log (v+1)-\log N)$. 
By the choice of $T$, we have $T+1 \geq 39 n^{2} \log \left(n^{2} \delta_{0}\right)$. By (3.24), (3.21) and (3.19),

$$
\begin{aligned}
& \lambda(\log (v+1)+\log N)+n \log (v+1)+\log N \\
& \quad \leq 2.91(\log N)\left(\log \left(n^{2} \delta_{0}\right)+(n+1) \log N\right)+n \log \left(n^{2} \delta_{0}\right)+\left(n^{2}+1\right) \log N \\
& \quad \leq 2.91(6.22 n(n+1)+1) \log \left(n^{2} \delta_{0}\right) \log N+n \log \left(n^{2} \delta_{0}\right)+\left(n^{2}+1\right) \log N \\
& \quad \leq c \cdot 39 n^{2} \log \left(n^{2} \delta_{0}\right) \log N
\end{aligned}
$$

with

$$
c=\frac{2.91(6.22 \cdot 1.5+0.25)+0.5 / 10.75+(1+0.25) / \log 4}{39} \leq 0.74
$$

(use $n \geq 2$ and (3.18)). Let

$$
f(t)=\frac{N}{t}\left(\frac{\log t}{\log N}-0.74\right) \log N .
$$

Then

$$
\theta^{-1}>\frac{39 n^{2} f(p) \log \left(n^{2} \delta_{0}\right)}{N v}
$$

We remark that $f(t)$ has a single stationary point on $[0,+\infty]$ which is a local maximum. Since $p \in\left[N^{3 / 4}, N\right]$, we have $f(p) \geq \min \left\{f\left(N^{3 / 4}\right), f(N)\right\}$. Moreover, by (3.18),

$$
f\left(N^{3 / 4}\right) \geq e^{10.75 / 4}(3 / 4-0.74) \cdot 10.75>1
$$

and $f(N) \geq(1-0.74) \cdot 10.75>1$. Thus $f(p)>1$. Using $(3.21)$, we finally obtain $\theta<\frac{N v}{39 n^{2} \log \left(n^{2} \delta_{0}\right)} \leq \frac{n^{2} N^{n+1} \delta_{0}}{39 n^{2} \log \left(n^{2} \delta_{0}\right)}=\delta_{0} n^{2}\left(39 n^{2} \log \left(n^{2} \delta_{0}\right)\right)^{(n+1)(k+1)-1}=\theta$.

This contradiction shows that the assumption (3.23) cannot hold. Thus we have:

$$
H\left(W^{\prime} ; v\right)<H\left(V^{\prime} ; v\right) .
$$

Equivalently, there exists a homogeneous polynomial $F$ of degree $v$ which vanishes on $W^{\prime}$ but not on $V^{\prime}$. The varieties are defined over the rationals, so we can assume $F \in \mathbb{Q}[\boldsymbol{x}]$. Since $F$ does not vanish on $V^{\prime}$, there exists a prime number $p \in \wp$ such that $F$ does not vanish on $[p] V$. Let $Z$ be the zero set of $F\left(x^{p}\right)=0$. Then $V \nsubseteq Z$ and $V\left(\theta^{-1}\right) \subseteq W \subseteq Z$. We have

$$
\operatorname{deg}(Z) \leq N \operatorname{deg} F \leq N v \leq n^{2} N^{n+1} \delta_{0}=\delta_{0} n^{2}\left(39 n^{2} \log \left(n^{2} \delta_{0}\right)\right)^{(n+1)(k+1)}
$$

as required. 


\section{Distribution of the small points}

A geometric reduction process, close to that of [Amo-Via], applied to each variety involved, allows us to prove the main result of this article using Theorem 1.2.

Theorem 1.3. Let $V_{0} \subseteq V_{1}$ be subvarieties of $\mathbb{G}_{\mathrm{m}}^{n}$, defined over $\mathbb{Q}$, of codimensions $k_{0}$ and $k_{1}$ respectively. Assume that $V_{0}$ is $\mathbb{Q}$-irreducible. Let

$$
\theta=\delta\left(V_{1}\right)\left(935 n^{5} \log \left(n^{2} \delta\left(V_{1}\right)\right)\right)^{\left(k_{0}-k_{1}+1\right)\left(k_{0}+1\right)(n+1)} .
$$

Then,

- either there exists a $\mathbb{Q}$-irreducible $B$ union of torsion varieties such that $V_{0} \subseteq$ $B \subseteq V_{1}$ and $\delta_{0}(B) \leq \theta$,

- or there exists a hypersurface $Z$ defined over $\mathbb{Q}$ of degree at most $\theta$ such that $V_{0} \nsubseteq Z$ and $V_{0}\left(\theta^{-1}\right) \subseteq Z$.

Proof. Theorem 1.3 is analogue to Theorem 2.2 of [Amo-Via]. The proof is similar. Let us give the details.

We simply denote $\delta=\delta\left(V_{1}\right)$. By contradiction, we suppose that the conclusion of Theorem 1.3 does not hold. Thus

$V_{0}$ is not contained in any union $B \subseteq V_{1}$ of proper torsion varieties with $\delta_{0}(B) \leq \theta$

and

Each hypersurface $Z$ defined over $\mathbb{Q}$, of degree $\leq \theta$, with $V_{0}\left(\theta^{-1}\right) \subseteq Z$ contains $V_{0}$.

For $r \in\left\{0, \ldots, k_{0}-k_{1}+1\right\}$ we define

$$
D_{r}=\delta\left(935 n^{5} \log \left(n^{2} \delta\right)\right)^{r\left(k_{0}+1\right)(n+1)} .
$$

Since $r \leq k_{0}-k_{1}+1$, we have $D_{r} \leq \theta$. Using an inductive process on $r$, we are going to construct a chain of varieties

$$
X_{0} \supseteq \cdots \supseteq X_{r} \supseteq X_{r+1} \supseteq \cdots \supseteq X_{k_{0}-k_{1}+1}
$$

defined over $\mathbb{Q}$ which satisfy:

\section{Claim.}

i) $V_{0} \subseteq X_{r}$.

ii) Each $\mathbb{Q}$-irreducible component of $X_{r}$ containing $V_{0}$ has codimension $\geq r+k_{1}$.

iii) $\delta\left(X_{r}\right) \leq D_{r}$. 
Theorem 1.3 is proved if we show the claim for $r=k_{0}-k_{1}+1$. Indeed, by i) there exists a $\mathbb{Q}$-irreducible component $W$ of $X_{k_{0}-k_{1}+1}$ which contains $V_{0}$. By ii) codim $W \geq k_{0}+1$. This gives a contradiction.

We now define $X_{r}$ and prove our claim by induction on $r$.

- For $r=0$, we simply choose $X_{0}=V_{1}$.

- We assume that our claim holds for some $r \in\left\{0, \ldots, k_{0}-k_{1}\right\}$ and we prove that it holds for $r+1$, as well. Since $V_{0} \subseteq X_{r}$, there exists at least one $\mathbb{Q}$ irreducible component of $X_{r}$ which contains $V_{0}$. Let $1 \leq s \leq t$ be integers and let $W_{1}, \ldots, W_{s}, W_{s+1}, \ldots, W_{t}$ be the $\mathbb{Q}$-irreducible components of $X_{r}$. We enumerate these components so that

$$
V_{0} \subseteq W_{j} \quad \text { if and only if } j=1, \ldots, s .
$$

Assertion ii) of our claim for $r$ implies that $r+k_{1} \leq \operatorname{codim}\left(W_{j}\right) \leq k_{0}$, for $j=$ $1, \ldots, s$.

Let $j \in\{1, \ldots, s\}$. Since $\delta\left(X_{r}\right) \leq D_{r}$, the variety $W_{j}$ is a $\mathbb{Q}$-irreducible component of an intersection of hypersurfaces defined over $\mathbb{Q}$ of degree $\leq D_{r}$. Thus $\delta_{0}\left(W_{j}\right) \leq D_{r} \leq \theta$. Moreover

$$
V_{0} \subseteq W_{j} \subseteq X_{r} \subseteq X_{0}=V_{1} .
$$

By assumption (4.25), $W_{j}$ is not a union of torsion varieties.

Let

$$
\theta_{0}=D_{r}\left(52 n^{2} \log \left(n^{2} D_{r}\right)\right)^{(n+1)\left(k_{0}+1\right)} .
$$

In view of Theorem 1.2 , the set $W_{j}\left(\theta_{0}^{-1}\right)$ is contained in a hypersurface $Z_{j}$ defined over $\mathbb{Q}$ which does not contain $W_{j}$ and such that $\operatorname{deg} Z_{j} \leq \theta_{0}$. We show that $\theta_{0} \leq D_{r+1}$. For this we need an upper bound for $\log \left(n^{2} D_{r}\right)$. Using $\log x<\sqrt{x}$ for $x>0$, we obtain

$$
\begin{aligned}
D_{r} & =\delta\left(935 n^{5} \log \left(n^{2} \delta\right)\right)^{r\left(k_{0}+1\right)(n+1)} \leq \delta\left(935 n^{5} \cdot n \delta\right)^{r\left(k_{0}+1\right)(n+1)} \\
& \leq \delta\left(935 n^{6} \delta\right)^{n(n+1)^{2}} .
\end{aligned}
$$

We have $n^{2} \leq n^{n^{3} / 4}, n(n+1)^{2} \leq(9 / 4) n^{3}$ and $935 \leq n^{(\log 935) / \log 2}$. Thus $n^{2} D_{r} \leq$ $\left(n^{2} \delta\right)^{c n^{3}}$ with

We deduce

$$
c=\frac{1}{8}+\frac{9}{4} \cdot \frac{1}{2}\left(\frac{\log 935}{\log 2}+6\right)<17.98 .
$$

$$
\begin{aligned}
\theta_{0} & \leq D_{r}\left(52 n^{2} \cdot 17.98 n^{3} \log \left(n^{2} \delta\right)\right)^{(n+1)\left(k_{0}+1\right)} \\
& \leq D_{r}\left(935 n^{5} \log \left(n^{2} \delta\right)\right)^{(n+1)\left(k_{0}+1\right)} \\
& =D_{r+1}
\end{aligned}
$$


Since $V_{0} \subseteq W_{j}$

$$
V_{0}\left(\theta_{0}^{-1}\right) \subseteq W_{j}\left(\theta_{0}^{-1}\right) \subseteq Z_{j} .
$$

As deg $Z_{j} \leq \theta_{0} \leq D_{r+1} \leq \theta$, relation (4.26) implies that $V_{0} \subseteq Z_{j}$. Thus, for $j=1, \ldots, s$ we have $V_{0} \subseteq Z_{j}$ and

$$
V_{0} \subseteq \bigcap_{j=1}^{s} Z_{j} .
$$

Let

$$
X_{r+1}=X_{r} \cap Z_{1} \cap \cdots \cap Z_{s} .
$$

Then $V_{0} \subseteq X_{r+1} \subseteq X_{r}$.

Recall that $\operatorname{deg} Z_{j} \leq \theta_{0} \leq D_{r+1}$. Then

$$
\delta\left(X_{r+1}\right) \leq \max \left\{\delta\left(X_{r}\right), D_{r+1}\right\} \leq \max \left\{D_{r}, D_{r+1}\right\}=D_{r+1} .
$$

We decompose

$$
X_{r+1}=W_{1}^{\prime} \cup \cdots \cup W_{s}^{\prime} \cup W_{s+1}^{\prime} \cup \cdots \cup W_{t}^{\prime},
$$

where $W_{j}^{\prime}=W_{j} \cap Z_{1} \cap \cdots \cap Z_{s}$.

Let $j \in\{1, \ldots, s\}$. Since $W_{j} \nsubseteq Z_{j}$, every $\mathbb{Q}$-irreducible component of $W_{j}^{\prime}$ has codimension $\geq \operatorname{codim}\left(W_{j}\right)+1 \geq r+1+k_{1}$.

Let $j \in\{s+1, \ldots, t\}$. Since $V_{0} \nsubseteq W_{j}$, the variety $V_{0}$ is not contained in any $\mathbb{Q}$-irreducible component of $W_{j}^{\prime}$.

We conclude that $X_{r+1}$ satisfies our claim for $r+1$.

\section{Proofs of Theorem 1.1 and of the corollaries}

Theorem 1.1 becomes a corollary of Theorem 1.3:

Proof of Theorem 1.1. Let

$$
\theta=\delta(V)\left(935 n^{5} \log \left(n^{2} \delta(V)\right)\right)^{(d+1)(n+1)^{2}} .
$$

We have to show that $V^{*}\left(\theta^{-1}\right)=\emptyset$. Let $V_{0}$ be one of the finitely many $\mathbb{Q}$-irreducible components of $\overline{V\left(\theta^{-1}\right)}$. Then $\overline{V_{0}\left(\theta^{-1}\right)}=V_{0}$. Apply Theorem 1.3 to $V_{0}$ and $V_{1}=V$. We have $k_{0} \leq n$ and $k_{1}=n-d$. Thus

$$
\left(k_{0}-k_{1}+1\right)\left(k_{0}+1\right)(n+1) \leq(d+1)(n+1)^{2} .
$$

Since $V\left(\theta^{-1}\right)$ is dense in $V_{0}$, the first assertion of Theorem 1.3 must hold. So $\overline{V_{0}\left(\theta^{-1}\right)}$ is contained in a union of torsion varieties $B \subseteq V$. Varying $V_{0}$ over all components of $\overline{V\left(\theta^{-1}\right)}$, we conclude that $\overline{V\left(\theta^{-1}\right)} \subseteq B$ where $B \subseteq V$ is a union of torsion varieties. Thus $V^{*}\left(\theta^{-1}\right)=\emptyset$. 
On the one hand, Theorem 1.1 tells us that the small points of $V$ are contained in the union $V^{u}$ of torsion varieties included in $V$. On the other hand, the torsion is dense in a torsion varieties and $V^{u}$ is a finite union of the maximal torsion varieties of $V$. Thus, the closure of the small points must be $V^{u}$. In [Amo-Via], Corollary 5.3, we estimate the sum of the degrees of these maximal torsion varieties. This is the line of

Proof of Corollary 1.4. Let $V^{u}=B_{1} \cup \cdots \cup B_{t}$ where $B_{j}$ are the maximal torsion varieties of $V$. By [Amo-Via], Corollary 5.3, $\delta_{0}\left(B_{j}\right) \leq \theta^{\prime}$ and

$$
\sum_{j=1}^{t} \theta^{\prime \operatorname{dim}\left(B_{j}\right)} \operatorname{deg}\left(B_{j}\right) \leq \theta^{\prime n}
$$

where $\theta^{\prime} \leq \theta$. Since $V^{*}=V \backslash V^{u}$, Theorem 1.1 shows that

$$
V\left(\theta^{-1}\right) \subseteq V^{u}=B_{1} \cup \cdots \cup B_{t} .
$$

In addition

$$
V^{u}=\overline{V(0)} \subseteq \overline{V\left(\theta^{-1}\right)}
$$

Let $V \subseteq \mathbb{G}_{\mathrm{m}}^{n}$ be a $\mathbb{Q}$-irreducible subvariety which is not contained in any union of proper torsion varieties. As remarked in the introduction, Theorem 1.1 implies a lower bound for the essential minimum. The slightly better lower bound of Corollary 1.5 is obtained directly from Theorem 1.3.

Proof of Corollary 1.5. Choose a hypersurface $Z$ defined over $\mathbb{Q}$ containing $V$ of minimal degree $\omega(V)$. The result follows choosing $V_{0}=V, V_{1}=Z, k_{0}=k$ and $k_{1}=1$ in Theorem 1.3.

Finally, we prove the lower bound for the product of the heights of multiplicatively independent algebraic numbers announced in the introduction in Corollary 1.6.

Proof of Corollary 1.6. We reorder $\alpha_{1}, \ldots, \alpha_{n}$ in such a way that $h\left(\alpha_{1}\right) \leq \cdots \leq$ $h\left(\alpha_{n}\right)$. Let $A_{i}=\left[2 h\left(\alpha_{i}\right) / h\left(\alpha_{1}\right)\right]$ and choose algebraic numbers $\beta_{1}, \ldots, \beta_{n}$ such that $\beta_{i}^{A_{i}}=\alpha_{i}$. We apply Corollary 1.5 to the 0 -dimensional variety $V$ of degree $[\mathbb{Q}(\boldsymbol{\beta}): \mathbb{Q}]$, consisting of the conjugates of $\boldsymbol{\beta}=\left(\beta_{1}, \ldots, \beta_{n}\right)$. We have

$$
\hat{\mu}^{\mathrm{ess}}(V)=h(\boldsymbol{\beta}) \leq \sum_{i} A_{i}^{-1} h\left(\alpha_{i}\right) \leq n h\left(\alpha_{1}\right) .
$$

By the bound (2.4) of Chardin, we deduce

$$
\begin{aligned}
\omega(V) & \leq n[\mathbb{Q}(\boldsymbol{\beta}): \mathbb{Q}]^{1 / n} \\
& \leq n\left(D A_{1} \ldots A_{n}\right)^{1 / n} \\
& \leq 2 n\left(h\left(\alpha_{1}\right) \ldots h\left(\alpha_{n}\right)\right)^{1 / n} h\left(\alpha_{1}\right)^{-1} D^{1 / n}
\end{aligned}
$$


In view of the upper bound for the essential minimum and in view of Corollary 1.5 we obtain

$$
\begin{aligned}
n h\left(\alpha_{1}\right) & \geq \hat{\mu}^{\text {ess }}(V) \\
& \geq(2 n)^{-1}\left(h\left(\alpha_{1}\right) \ldots h\left(\alpha_{n}\right)\right)^{-1 / n} h\left(\alpha_{1}\right) D^{-1 / n}\left(935 n^{5} \log \left(n^{2} \omega(V)\right)\right)^{-n(n+1)^{2}}
\end{aligned}
$$

or equivalently

$$
h\left(\alpha_{1}\right) \ldots h\left(\alpha_{n}\right) \geq D^{-1}\left(2 n^{2}\right)^{-n}\left(935 n^{5} \log \left(n^{2} \omega(V)\right)\right)^{-n^{2}(n+1)^{2}} .
$$

To conclude the proof, we use an effective lower bound for the height due to P. Voutier. Note that $\alpha_{1}$ is not a root of unity. By [Vou], Corollary $2, h\left(\alpha_{1}\right) \geq 2 D^{-1} \log (3 D)^{-3}$. Moreover we can clearly assume $D \geq 2$ and

$$
h\left(\alpha_{1}\right) \ldots h\left(\alpha_{n}\right) \leq D^{-1}(n \log (3 D))^{-3 n} .
$$

Thus,

$$
\omega(V) \leq 2 n \cdot D^{-1 / n}(n \log (3 D))^{-3} \cdot \frac{1}{2} D \log (3 D)^{3} \cdot D^{1 / n}=n^{-2} D
$$

and (using $\left(2 n^{2}\right)^{1 / n(n+1)^{2}} \cdot 935 \leq 8^{1 / 18} \cdot 935 \leq 1050$ for $n \geq 2$ )

$$
\begin{aligned}
\left(2 n^{2}\right)^{n}\left(935 n^{5} \log \left(n^{2} \omega(V)\right)\right)^{n^{2}(n+1)^{2}} & \leq\left(2 n^{2}\right)^{n}\left(935 n^{5} \log D\right)^{n^{2}(n+1)^{2}} \\
& \leq\left(1050 n^{5} \log (3 D)\right)^{n^{2}(n+1)^{2}} .
\end{aligned}
$$

\section{Appendix}

The following appendix by M. Chardin and P. Philippon contains two results. The first one is an extension of the lower bound for the Hilbert function proved in [Cha-Phi]. This result is crucial in the proof of Proposition 3.3. The second result in this appendix deals with a filtration of invariants starting with $\omega$ and ending with $\delta_{0}$. Let $V \subset \mathbb{P}^{n}$ be a $K$-irreducible variety of codimension $k$ defined by a homogeneous prime ideal $I \subseteq A=\mathbb{K}\left[x_{0}, \ldots, x_{n}\right]$. Let $1 \leq r \leq k$. Philippon (see [Phi], Corollary 6) defines $\delta_{r}^{\prime}(I)$ as the minimal degree $\delta$ such that there exist homogeneous polynomials $f_{1}, \ldots, f_{r} \in A$ of degree $\delta$ which form a regular sequence in $I A_{I}$. Thus $\delta_{r}^{\prime}(I)$ is the minimal degree $\delta$ such that there exists an intersection $X$ of hypersurfaces defined over $K$ of degree $\leq \delta$ containing $V$ and of local codimension $\geq r$ at $V$. The proof of Corollary 2.3 shows that it is not restrictive to require also that all hypersurfaces are defined over $\overline{\mathbb{Q}}$. Thus $\delta_{1}^{\prime}(I)=\omega(V)$ and $\delta_{k}^{\prime}(I)=\delta_{0}(V)$. In addition, one can show that $V$ is an isolated component of an intersection of $k$ hypersurfaces of 
degree $\delta_{1}^{\prime}(I), \ldots, \delta_{k}^{\prime}(I)$. Thus, by Bézout's theorem, $\operatorname{deg}(V) \leq \delta_{1}^{\prime}(I) \ldots \delta_{k}^{\prime}(I)$. In the second part of the appendix, M. Chardin and P. Philippon prove that there exist hypersurfaces $Z_{1}, \ldots, Z_{k}$ of degree $d_{1}, \ldots, d_{k}$ such that $V$ is an isolated component of $Z_{1} \cap \cdots \cap Z_{k}$ and

$$
\left(n^{k(k+1) / 2} 2^{n k(k-1)}\right)^{-1} d_{1} \ldots d_{k} \leq \operatorname{deg}(V) \leq d_{1} \ldots d_{k} .
$$

Obviously, by definition $\delta_{r}^{\prime} \leq d_{r}$. In addition, since $\operatorname{deg}(V) \leq \delta_{1}^{\prime}(I) \ldots \delta_{k}^{\prime}(I)$, we deduce

$$
\left(n^{k(k+1) / 2} 2^{n k(k-1)}\right)^{-1} \delta_{1}^{\prime}(I) \ldots \delta_{k}^{\prime}(I) \leq \operatorname{deg}(I) \leq \delta_{1}^{\prime}(I) \ldots \delta_{k}^{\prime}(I) .
$$

Even if these inequalities are not needed here, we believe that they will be useful.

\section{Complément à [Cha-Phi]}

Par M. Chardin et P. Philippon

6.1. Extension de la minoration de fonction de Hilbert. Dans l'énoncé suivant, nous utilisons la notion de modules et schémas $(m, \mathfrak{b})$-parfaits telle qu'introduite dans [Cha-Phi]. Rappelons que dans cette propriété $m$ est un entier et $\mathfrak{b}$ est un idéal homogène de l'anneau de base (supposé gradué). En particulier, l'espace projectif $\mathbb{P}^{n}$ est 0 -régulier et son anneau de coordonnées $A=\mathbf{k}\left[x_{0}, \ldots, x_{n}\right]$ est $(0, A)$-régulier (en tant que $A$-module).

Théorème 6.1. Soient $V_{1}, \ldots, V_{s}$ des sous-schémas de $\mathbb{P}^{n}$, équi-dimensionnels de même dimension $D$ et de supports deux à deux distincts. Notons $\mathfrak{b}_{1}, \ldots, \mathfrak{b}_{s}$ des idéaux homogènes de l'anneau de coordonnées $A=\mathbf{k}\left[x_{0}, \ldots, x_{n}\right]$. On suppose que $V_{i}$ est $\left(m_{i}, \mathfrak{b}_{i}\right)$-parfait pour $i=1, \ldots, s$ et on note $V$ un sous-schéma de dimension $D$ contenu dans $V_{1} \cup \cdots \cup V_{s}$. Alors on a

$$
\mathscr{H}(V, v) \geq \operatorname{deg}(V)\left(\begin{array}{c}
v+D-m \\
D
\end{array}\right)
$$

dès que $v>m:=m_{1}+\cdots+m_{s}+s-1$.

Nota Bene - Posons $\delta_{0}(V)$ le plus petit entier tel que $V$ soit composante d'une intersection de $n-D$ formes de degré au plus $\delta_{0}$. On sait que $m_{i} \leq(n-D)\left(\delta_{0}\left(V_{i}\right)-1\right)$ et on a donc dans l'énoncé ci-dessus :

$$
m \leq(n-D)\left(\delta_{0}\left(V_{1}\right)+\cdots+\delta_{0}\left(V_{s}\right)-s\right)+s-1 .
$$

Démonstration. On procède par récurrence sur $D$, on note $A=\mathbf{k}\left[x_{0}, \ldots, x_{n}\right]$ et $I_{1}, \ldots, I_{s}$ les idéaux des $V_{i}$. Pour $D=0$ on sait que le $A$-module $A / I_{i}$ est $\left(m_{i}, \mathfrak{b}_{i}\right)$ parfait et donc $m_{i}$-régulier d'après [Cha-Phi], proposition 3. D'après le théorème 2.4 
de [Con-Her] (appliqué avec $M=A /\left(I_{1} \cap \cdots \cap I_{i-1}\right)$ et $A / I_{i}$ qui est de dimension 1) on sait que la régularité de $I_{i} /\left(I_{1} \cap \cdots \cap I_{i}\right)$ est majorée par la somme de la régularité de $A /\left(I_{1} \cap \cdots \cap I_{i-1}\right)$ et de celle de $I_{i}$ (qui est égale à celle de $A / I_{i}$ plus 1 ). De plus, la régularité de $A /\left(I_{1} \cap \cdots \cap I_{i}\right)$ est le maximum de celle de $I_{i} /\left(I_{1} \cap \cdots \cap I_{i}\right)$ et de celle de $A / I_{i}$, d'où les inégalités

$$
\begin{aligned}
\operatorname{reg}\left(A /\left(I_{1} \cap \cdots \cap I_{i}\right)\right) & \leq \max \left(\operatorname{reg}\left(I_{i} /\left(I_{1} \cap \cdots \cap I_{i}\right) ; \operatorname{reg}\left(A / I_{i}\right)\right)\right. \\
& \leq \operatorname{reg}\left(A /\left(I_{1} \cap \cdots \cap I_{i-1}\right)+\operatorname{reg}\left(A / I_{i}\right)+1 .\right.
\end{aligned}
$$

Comme la régularité de $A / I_{i}$ est majorée par $m_{i}$ on obtient par téléscopage que la régularité de $A /\left(I_{1} \cap \cdots \cap I_{s}\right)$ est majorée par $m_{1}+\cdots+m_{s}+s-1$. L'idéal $J$ de $V$ contient $I_{1} \cap \cdots \cap I_{s}$ et $A / J$ a même dimension $D$, la minoration cherchée résulte alors de [Cha-Phi], proposition 4 , dans ce cas.

Pour passer de $D-1$ à $D$ on intersecte, comme dans loc. cit., $V$ par une forme linéaire $x$ assez générale de sorte que pour tout $i, j \in\{1, \ldots, s\}$ on ait $\operatorname{dim}\left(V_{i} \cap\right.$ $\left.V_{j} \cap \mathcal{Z}(x)\right)<D-1$ et $\operatorname{dim}\left(V_{i} \cap \mathcal{Z}\left(\mathfrak{b}_{i}+x A\right)\right)<D-1$. On note $W_{i}$ la partie de dimension $D-1$ de $V_{i} \cap \mathcal{Z}(x)$ et on vérifie que $W_{i}$ est $\left(m_{i}, b_{i} \mathfrak{b}_{i}\right)$-parfait pour un $b_{i} \in A$ convenable. De plus les $W_{i}$ sont deux à deux distincts, en posant $W=W_{1} \cup \cdots \cup W_{s}$ on a $\operatorname{deg}(W)=\operatorname{deg}(V)$ et

$$
\mathscr{H}(V, v)-\mathscr{H}(V, v-1)=\mathscr{H}(V \cap \mathcal{Z}(x), v) \geq \mathscr{H}(W, v) .
$$

L'hypothèse de récurrence entraîne donc

$$
\mathscr{H}(V, v)-\mathscr{H}(V, v-1) \geq \operatorname{deg}(V)\left(\begin{array}{c}
v+D-1-m \\
D-1
\end{array}\right)
$$

puis la minoration voulue par intégration finie.

6.2. Complément à l'interpolation : estimations du degré. Dans le théorème 2 de [Cha-Phi], on vérifie de plus :

$$
\left(n^{r(r+1) / 2} 2^{n r(r-1)}\right)^{-1} d_{1} \ldots d_{r} \leq \operatorname{deg}(X) \leq d_{1} \ldots d_{r} .
$$

La majoration $\operatorname{deg}(X) \leq d_{1} \ldots d_{r}$ est une conséquence du théorème de Bézout. Pour l'autre inégalité, on peut en fait établir les propriétés supplémentaires suivantes, à annexer à celles $(1)_{i},(2)_{i}$ et $(3)_{i}$ du théorème 2 de [Cha-Phi]. Pour $i=1, \ldots, r$ on pose $c_{i}=\left(n^{i(i+1) / 2} 2^{n i(i-1)}\right)^{-1}$ et cette propriété s'énonce :

(4) $i$ pour toute composante $Y$ de $X_{i}$ on a $\mathscr{H}\left(Y, d_{i}-1\right) \geq c_{i} d_{1} \ldots d_{i}\left(\begin{array}{c}d_{i}+n-i \\ n-i\end{array}\right)$. Et en particulier $\operatorname{deg}\left(X_{i}\right) \geq \operatorname{deg}(Y) \geq c_{i} d_{1} \ldots d_{i} \geq c_{i} \operatorname{deg}\left(X_{i}\right)$.

La démonstration se fait dans la récurrence sur $i=1, \ldots, r$ et pour $i=r$ on a bien $\operatorname{deg}(X) \geq c_{r} d_{1} \ldots d_{r} \geq c_{r} \operatorname{deg}(X) \operatorname{car} X_{r}=X$. Le cas $i=1$ résulte déjà de $(2)_{1}\left(c_{1}=1 / n\right)$ et pour la récurrence l' argument à ajouter est le suivant $(1<i \leq r)$ : 
Comme $X \subset Y$ on a, par (2) $)_{i}$ et (4) $)_{i-1}$,

$$
\begin{aligned}
\mathscr{H}\left(Y, d_{i}-1\right) & \geq \mathscr{H}\left(X, d_{i}-1\right) \\
& \geq c(n, i)^{-1} \operatorname{deg}\left(X_{i-1}\right) d_{i}\left(\begin{array}{c}
d_{i}+n-i \\
n-i
\end{array}\right) \\
& \geq c(n, i)^{-1} c_{i-1} d_{1} \ldots d_{i}\left(\begin{array}{c}
d_{i}+n-i \\
n-i
\end{array}\right) .
\end{aligned}
$$

Ce qui conclut car $c_{i} \leq c_{i-1} c(n, i)^{-1}$, vu que $c(n, i)=\frac{n !}{(n-i) !} 2^{(i-1)(2 n-i)} \leq$ $n^{i} 4^{n(i-1)}$.

\section{References}

[Amo-Dav] F. Amoroso and S. David, Le problème de Lehmer en dimension supérieure. J. Reine Angew. Math. 513 (1999), 145-179. Zbl 1011.11045 MR 1713323

[Amo-Dav] F. Amoroso and S. David, Minoration de la hauteur normalisée des hypersurfaces. Acta Arith. 92 (2000), no. 4, 340-366. Zbl 0948.11025 MR 1760242

[Amo-Dav] F. Amoroso and S. David, Densité des points à cordonnées multiplicativement indépendantes. Ramanujan J. 5 (2001), 237-246. Zbl 0996.11046 MR 1876697

[Amo-Dav] F. Amoroso and S. David, Minoration de la hauteur normalisée dans un tore. J. Inst. Math. Jussieu 2 (2003), no. 3, 335-381. Zbl 1041.11048 MR 1990219

[Amo-Dav] F. Amoroso and S. David, Distribution des points de petite hauteur dans les groupes multiplicatifs. Ann. Scuola Norm. Sup. Pisa Cl. Sci. (5) 3 (2004), no. 2, 325-348. Zbl 1150.11021 MR 2075986

[Amo-Via] F. Amoroso and E. Viada, Small points on subvarieties of a torus. Duke Math. J. 150 (2009), no. 3, 407-442. Zbl 05688238 MR 2582101

[Bom-Mas-Zan] E. Bombieri, D. Masser and U. Zannier, Intersecting a curve with algebraic subgroups of multiplicative groups. Internat. Math. Res. Notices 20 (1999), 1119-1140. Zbl 0938.11031 MR 1728021

[Bom-Vaa] E. Bombieri and J. Vaaler, On Siegel's lemma. Invent. Math. 73 (1983), 11-32. Zbl 0533.10030 MR 0707346

[Cha] M. Chardin, Une majoration de la fonction de Hilbert et ses conséquences pour l'interpolation algébrique. Bull. Soc. Math. France 117 (1988), 305-318. Zbl 0709.13007 MR 1020108

[Cha-Phi] M. Chardin and P. Philippon, Régularité et interpolation. J. Algebraic Geom. 8 (1999), no. 3, 471-481; erratum, ibid. 11 (2002), 599-600. Zbl 0962.13014 MR 1894934

[Con-Her] A. Conca and J. Herzog, Castelnuovo-Mumford regularity of products of ideals. Collect. Math. 54 (2003), 137-152. Zbl 1074.13004 MR 1995137 
[Dav-Phi] S. David and P. Philippon, Minorations des hauteurs normalisées des sousvariétés des tores. Ann. Scuola Norm. Sup. Pisa Cl. Sci. (4) 28 (1999), no. 3, 489-543; errata, ibid. 29 (2000), no. 3, 729-731. Zbl 1002.11055 MR 1736526

[Del] E. Delsinne, Le problème de Lehmer relatif en dimension supérieure. Ann. Sci. Éc. Norm. Supér (4) 42 (2009), no. 6, 981-1028. Zbl 05654770 MR 2567747

[Dob] E. Dobrowolski, On a question of Lehmer and the number of irreducible factors of a polynomial. Acta Arith. 34 (1979), 391-401. Zbl 0416.12001 MR 0543210

[Leh] D. H. Lehmer, Factorization of certain cyclotomic functions. Ann. of Math. 34 (1933), 461-479. Zbl 0007.19904 MR 1503118

[Phi] P. Philippon, Sur des hauteurs alternatives. III. J. Math. Pures Appl. (9) 74 (1995), no. 4, 345-365. Zbl 0878.11025 MR 1341770

[Phi] P. Philippon, Approximation algébrique des points dans les espaces projectifs. I. J. Number Theory 81 (2000), 234-253. Zbl 1096.11504 MR 1752252

[Ros-Sch] J. B. Rosser and L. Schoenfeld, Approximate formulas for some functions of prime numbers. Illinois J. Math. 6 (1962), 64-94. Zbl 0122.05001 MR 0137689

[Sch] W. M. Schmidt, Heights of points on subvarieties of $\mathbb{G}_{\mathrm{m}}^{n}$. In Number Theory 93-94, ed. by S. David, London Math. Soc. Lecture Note Ser. 235, Cambridge University Press, Cambridge 1996, 157-187. Zbl 0917.11023 MR 1628798

[Vou] P. Voutier, An effective lower bound for the height of algebraic numbers. Acta Arith. 74 (1996), no. 1, 81-95. Zbl 0838.11065 MR 1367580

[Zha] S. Zhang, Positive line bundles on arithmetic varieties. J. Amer. Math. Soc. 8 (1995), no. 1, 187-221. Zbl 0861.14018 MR 1254133

Received July 7, 2009

Francesco Amoroso, Université de Caen, Laboratoire de mathématiques, Campus II, BP 5186, 14032 Caen Cedex, France

E-mail: francesco.amoroso@math.unicaen.fr

Evelina Viada, Department of Mathematics, University of Basel, Rheinsprung 21, 4051 Basel, Switzerland

E-mail: evelina.viada@unibas.ch 